\title{
Affine frames, GMRA's, and the canonical dual
}

by

\author{
Marcin Bownik (Ann Arbor, MI) and ERIC WeBer (Laramie, WY)
}

\begin{abstract}
We show that if the canonical dual of an affine frame has the affine structure, with the same number of generators, then the core subspace $V_{0}$ is shift invariant. We demonstrate, however, that the converse is not true. We apply our results in the setting of oversampling affine frames, as well as in computing the period of a Riesz wavelet, answering in the affirmative a conjecture of Daubechies and Han. Additionally, we completely characterize when the canonical dual of a quasi-affine frame has the quasi-affine structure.
\end{abstract}

The study of affine systems in $L^{2}\left(\mathbb{R}^{d}\right)$ which gives rise to frames is currently under vigorous research. Such systems can have better properties than systems which are bases [16], and can be utilized in denoising [2]. Since frames can have many duals which yield reconstruction, from a practical print of view it is important to know what structure the duals have. In particular, one wishes to know if a given affine frame has any duals with the affine structure.

From a strictly theoretical viewpoint, one can ask if an affine frame generates a generalized multiresolution analysis (GMRA). A GMRA, as defined by Baggett, Medina, and Merrill [1], is a sequence $\left\{V_{j}: j \in \mathbb{Z}\right\}$ of closed subspaces of $L^{2}\left(\mathbb{R}^{d}\right)$ with the following five properties:

1. $V_{j} \subset V_{j+1}$

2. $D V_{j}=V_{j+1}$;

3. $\overline{\bigcup_{j \in \mathbb{Z}} V_{j}}=L^{2}\left(\mathbb{R}^{d}\right)$;

4. $\bigcap_{j \in \mathbb{Z}} V_{j}=\{0\}$;

5. $T_{z} V_{0} \subset V_{0}$ for all $z \in \mathbb{Z}^{d}$.

Here, $T_{z}$ is a translation operator given by $T_{z} f(x)=f(x-z)$ and $D$ is a dilation operator given by $D f(x)=|\operatorname{det} A|^{1 / 2} f(A x)$ for some $d \times d$ expansive integer-valued matrix $A$. In particular, for any $l \in \mathbb{Z}^{d}$ we have $D^{*} T_{l} D=T_{k}$ for some $k \in \mathbb{Z}^{d}$.

2000 Mathematics Subject Classification: Primary 42C40, 46N99.

Both authors were supported in part by grants from the National Science Foundation. 
Given a frame wavelet $\Psi$, we define a sequence of subspaces by

$$
V_{j}(\Psi):=\overline{\operatorname{span}}\left\{D^{n} T_{z} \psi: n<j, z \in \mathbb{Z}^{d}, \psi \in \Psi\right\} .
$$

Clearly, $\left\{V_{j}(\Psi): j \in \mathbb{Z}\right\}$ satisfies items 1,2 and 3 . However, there are examples of frame wavelets such that either 4 or 5 fails to hold.

Upon first inspection, it may seem that the conditions that the canonical dual of $\Psi$ is a frame wavelet and $\Psi$ generates a GMRA are independent. But in fact they are connected, though the purpose of this paper is to demonstrate that this connection is unlike the Riesz basis case. If $\Psi$ generates a Riesz basis, then condition 4 above holds. Condition 5 holds, however, if and only if the (unique) dual of $\left\{D^{n} T_{z} \psi: n \in \mathbb{Z}, z \in \mathbb{Z}^{d}, \psi \in \Psi\right\}$ is of the form $\left\{D^{n} T_{z} \phi: n \in \mathbb{Z}, z \in \mathbb{Z}^{d}, \phi \in \Phi\right\}$, with the cardinalities of the sets $\Psi$ and $\Phi$ being equal [15, 21, 22].

The obvious extension of this fact to frame wavelets is false, as described in the following two theorems.

TheOREm 1. Suppose $\Psi=\left\{\psi_{1}, \ldots, \psi_{r}\right\}$ is an affine frame such that the standard dual is again an affine frame with generators $\Phi=\left\{\phi_{1}, \ldots, \phi_{r}\right\}$. Then the core subspace

$$
V_{0}(\Psi):=\overline{\operatorname{span}}\left\{D^{n} T_{l} \psi_{i}: n<0, l \in \mathbb{Z}^{d}, i=1, \ldots, r\right\}
$$

is shift invariant.

THEOREM 2. (i) There exists an affine frame $\left\{D^{n} T_{l} \psi\right\}$ with an alternate dual $\left\{D^{n} T_{l} \eta\right\}$ but with $V_{0}(\psi)$ not shift invariant.

(ii) There exists an affine frame $\left\{D^{n} T_{l} \psi\right\}$ with no affine duals but with $V_{0}(\psi)$ shift invariant.

A complete characterization of when the canonical dual of a frame wavelet $\Psi$ is also a frame wavelet is unknown at this time. For quasi-affine frames, however, we announce the characterization below.

The quasi-affine system was introduced by Ron and Shen [24] to accommodate shift invariant space theory to affine frames. Define the operator $\widetilde{D}$ by renorming $D: \widetilde{D} f(x)=|\operatorname{det} A| f(A x)$. The quasi-affine system is given by

$$
\mathcal{U}^{q}=\left\{D^{n} T_{z}: n \geq 0, z \in \mathbb{Z}^{d}\right\} \cup\left\{T_{z} \widetilde{D}^{n}: n<0, z \in \mathbb{Z}^{d}\right\} .
$$

As with the affine system, if $\mathcal{U}^{q}(\Psi)$ is a frame, one wishes to know if any of its duals also has the form $\mathcal{U}^{q}(\Phi)$, and in particular, if the standard dual has the quasi-affine structure.

Theorem 3. Suppose $\mathcal{U}^{q}(\Psi)$ is a frame in $L^{2}\left(\mathbb{R}^{d}\right)$. The canonical dual is of the form $\mathcal{U}^{q}(\Phi)$ if and only if

$$
t_{q}(\xi):=\sum_{\psi \in \Psi} \sum_{j=0}^{\infty} \overline{\widehat{\psi}\left(A^{* j} \xi\right)} \widehat{\psi}\left(A^{* j}(\xi+2 \pi q)\right)=0 \quad \text { for a.e. } \xi
$$

for $q \in \mathbb{Z}^{d} \backslash A^{*} \mathbb{Z}^{d}$. 
This paper is organized as follows. We conclude this section with necessary definitions and preliminary results. In Section 1 we prove Theorems 1-3. In Section 2 we apply Theorem 1 to problems in oversampling of affine frames and computing the period of an affine frame. In particular, we provide a partial answer to a conjecture of Daubechies and Han regarding periods of frame wavelets by showing that a period of a dyadic Riesz wavelet is either 0 or $2^{J}$ for some non-negative integer $J$. Finally, in Section 3 we give a number of examples illustrating Theorem 2 .

A frame for a separable Hilbert space $\mathcal{H}$ is a collection of vectors $\left\{x_{j}\right\}_{j \in J}$, indexed by a countable set, such that there exist constants $0<C_{1} \leq C_{2}<\infty$ for which

$$
C_{1}\|v\|^{2} \leq \sum_{j \in \mathbb{J}}\left|\left\langle v, x_{j}\right\rangle\right|^{2} \leq C_{2}\|v\|^{2} \quad \text { for all } v \in \mathcal{H} .
$$

If only the upper bound holds in the above inequality (that is, $C_{1}=0$ ), then $\left\{x_{j}\right\}$ is said to be a Bessel sequence with a constant $C_{2}$.

The frame operator for a frame $\left\{x_{j}\right\}$, which is a bounded, positive, invertible operator, is given by

$$
S: \mathcal{H} \rightarrow \mathcal{H}, \quad v \mapsto \sum_{j \in \mathbb{J}}\left\langle v, x_{j}\right\rangle x_{j} .
$$

The frame $\left\{x_{j}\right\}$ is said to be tight if we can choose $C_{1}=C_{2}$; this is equivalent to $S=C_{1} I$, where $I$ is the identity operator. A second frame $\left\{y_{j}\right\}$ is dual to $\left\{x_{j}\right\}$ if

$$
v=\sum_{j \in \mathbb{J}}\left\langle v, y_{j}\right\rangle x_{j}
$$

at least one dual always exists, called the canonical (or standard) dual, given by $\left\{S^{-1} x_{j}\right\}$. In general, a frame has many duals; a dual which is not the canonical dual is called an alternate dual.

The affine system is $\mathcal{U}:=\left\{D^{n} T_{z}: n \in \mathbb{Z}, z \in \mathbb{Z}^{d}\right\}$. Following [14], the local commutant of the affine system, at $\psi$, is

$$
\mathcal{C}_{\psi}(\mathcal{U}):=\left\{B \in \mathcal{B}\left(L^{2}\left(\mathbb{R}^{d}\right)\right): B D^{n} T_{z} \psi=D^{n} T_{z} B \psi \forall D^{n} T_{z} \in \mathcal{U}\right\} .
$$

The canonical dual of an affine frame $\mathcal{U}(\Psi)$ is $\mathcal{U}(\Phi)$ if and only if

$$
\begin{aligned}
\left\{S^{-1} D^{n} T_{z} \psi_{i}: n \in \mathbb{Z}, z\right. & \left.\in \mathbb{Z}^{d}, i=1, \ldots, r\right\} \\
& =\left\{D^{n} T_{z} S^{-1} \psi_{i}: n \in \mathbb{Z}, z \in \mathbb{Z}^{d}, i=1, \ldots, r\right\} \\
& =\left\{D^{n} T_{z} \phi_{i}: n \in \mathbb{Z}, z \in \mathbb{Z}^{d}, i=1, \ldots, r\right\},
\end{aligned}
$$

where $S$ is the frame operator of $\mathcal{U}(\Psi)$. Equivalently, the canonical dual of $\mathcal{U}(\Psi)$ has the affine structure if and only if $S^{-1} \in \mathcal{C}_{\psi}(\mathcal{U})$ for all $\psi \in \Psi$.

If $W \subset L^{2}\left(\mathbb{R}^{d}\right)$ is a closed subspace, and $M$ is a $d \times d$ matrix, we say $W$ is $M \mathbb{Z}^{d}$-SI, or $M \mathbb{Z}^{d}$ shift invariant, if $T_{M z} W \subset W$ for all $z \in \mathbb{Z}^{d}$. In case $M$ is the identity matrix, we shall say that $W$ is shift invariant, or SI. 
Finally, in this paper, the Fourier transform is defined for $f \in L^{1}\left(\mathbb{R}^{d}\right)$ by

$$
\widehat{f}(\xi)=\int_{\mathbb{R}^{d}} f(x) e^{-i x \cdot \xi} d x .
$$

Given a measurable subset $K \subset \mathbb{R}^{d}$ we define the space $\check{L}^{2}(K)$, which is invariant under all translations, by

$$
\check{L}^{2}(K)=\left\{f \in L^{2}\left(\mathbb{R}^{d}\right): \operatorname{supp} \widehat{f} \subset K\right\} .
$$

1. Proofs of the main results. We set out to prove Theorem 1 .

Proof of Theorem 1. Define bounded operators $B_{0}$ and $B_{1}$ by

$$
\begin{aligned}
B_{0} & :=\sum_{i=1}^{r} \sum_{n<0} \sum_{z \in \mathbb{Z}^{d}}\left\langle\cdot, D^{n} T_{z} \phi_{i}\right\rangle D^{n} T_{z} \psi_{i}, \\
B_{1} & :=\sum_{i=1}^{r} \sum_{n \geq 0} \sum_{z \in \mathbb{Z}^{d}}\left\langle\cdot, D^{n} T_{z} \phi_{i}\right\rangle D^{n} T_{z} \psi_{i} .
\end{aligned}
$$

Note that $B_{0}+B_{1}=I$, the identity operator on $L^{2}\left(\mathbb{R}^{d}\right)$. A trivial exercise shows that $B_{1}$ commutes with the operators $T_{z}$ for all $z \in \mathbb{Z}^{d}$. Therefore, the closure of the range of $B_{0}$ is invariant under $T_{z}$.

Thus, we only need to show that $V_{0}(\Psi)=\overline{B_{0}\left(L^{2}\left(\mathbb{R}^{d}\right)\right)}$. Clearly, by definition of $V_{0}(\Psi)$ and $B_{0}$, we see that $B_{0}\left(L^{2}\left(\mathbb{R}^{d}\right)\right) \subset V_{0}(\Psi)$. We claim that $B_{0}\left(L^{2}\left(\mathbb{R}^{d}\right)\right)^{\perp} \subset V_{0}(\Psi)^{\perp}$. Let $f \in B_{0}\left(L^{2}\left(\mathbb{R}^{d}\right)\right)^{\perp}$; thus $\left\langle B_{0} g, f\right\rangle=0$ for $g \in L^{2}\left(\mathbb{R}^{d}\right)$.

Since $\Phi$ is the canonical dual wavelet frame of $\Psi$, we can choose $g \in$ $L^{2}\left(\mathbb{R}^{d}\right)$ such that $\left\langle g, D^{n} T_{z} \phi_{i}\right\rangle=\left\langle f, D^{n} T_{z} \psi_{i}\right\rangle$ for all $n \in \mathbb{Z}, z \in \mathbb{Z}^{d}$ and $i=1, \ldots, r$. Hence,

$$
\begin{aligned}
\left\langle B_{0} g, f\right\rangle & =\left\langle\sum_{i=1}^{r} \sum_{n<0} \sum_{z \in \mathbb{Z}^{d}}\left\langle g, D^{n} T_{z} \phi_{i}\right\rangle D^{n} T_{z} \psi_{i}, f\right\rangle \\
& =\sum_{i=1}^{r} \sum_{n<0} \sum_{z \in \mathbb{Z}^{d}}\left\langle g, D^{n} T_{z} \phi_{i}\right\rangle\left\langle D^{n} T_{z} \psi_{i}, f\right\rangle \\
& =\sum_{i=1}^{r} \sum_{n<0} \sum_{z \in \mathbb{Z}^{d}}\left|\left\langle f, D^{n} T_{z} \psi_{i}\right\rangle\right|^{2}=0 .
\end{aligned}
$$

Our claim now follows.

Two frame wavelets $\Psi$ and $\Phi$ which have shift invariant core spaces are called core equivalent if there exists a unitary operator $U: V_{0}(\Psi) \rightarrow V_{0}(\Phi)$ such that $U T_{z}=T_{z} U$ for all $z \in \mathbb{Z}^{d}$. The following is a corollary to the proof of Theorem 1. 
Corollary 1. If $\Phi$ is the standard dual of $\Psi$, then $\Phi$ and $\Psi$ are core equivalent.

Proof. We can consider the operator $B_{0}$ as defined above as an operator from $V_{0}(\Phi)$ to $V_{0}(\Psi)$. Let $B_{0}=U\left|B_{0}\right|$ be the polar decomposition of $B_{0}$, where $U$ is the partial isometry from the range of $\left|B_{0}\right|$ into $V_{0}(\Psi)$. Note that $U$ has the property that $U T_{z}=T_{z} U$.

The proof of Theorem 1 demonstrated that $B_{0}$ has dense range in $V_{0}(\Psi)$. By symmetry, $B_{0}^{*}$ has dense range in $V_{0}(\Phi)$. Therefore, $\left|B_{0}\right|$ has dense range in $V_{0}(\Phi)$ and the kernel of $U$ is trivial. Hence, $U$ can be extended to an isometry $\widetilde{U}: V_{0}(\Phi) \rightarrow V_{0}(\Psi)$ with trivial kernel. It follows that $\widetilde{U}$ is the necessary intertwining unitary.

In the proof of Theorem 2 we will use the following two standard results. Theorem 4 gives a sufficient condition for an affine system to be a Bessel sequence. Its proof can be found in [20, Theorem 13.0.1] or in the proof of [13, Lemma 3.4]. Lemma 1 is a basic perturbation result for frames, which can be found in [9, Corollary 2.7] or [17, Theorem 3].

THEOREM 4. Suppose that $f \in L^{2}(\mathbb{R})$ is such that $\widehat{f} \in L^{\infty}(\mathbb{R})$ and

$$
\widehat{f}(\xi)= \begin{cases}O\left(|\xi|^{\delta}\right) & \text { as } \xi \rightarrow 0, \\ O\left(|\xi|^{-1 / 2-\delta}\right) & \text { as }|\xi| \rightarrow \infty,\end{cases}
$$

for some $\delta>0$. Then the affine system $\left\{D^{n} T_{l} f\right\}$ is a Bessel sequence, where $D f(x)=\sqrt{2} f(2 x)$.

Lemma 1. Suppose $\mathcal{H}$ is a Hilbert space, $\left\{f_{i}\right\} \subset \mathcal{H}$ is a frame with constants $C_{1}$ and $C_{2}$, and $\left\{g_{i}\right\} \subset \mathcal{H}$ is a Bessel sequence with constant $C_{0}$. If $C_{0}<C_{1}$, then $\left\{f_{i}+g_{i}\right\}$ is a frame with constants $\left(\left(C_{1}\right)^{2}-\left(C_{0}\right)^{2}\right)^{1 / 2}$ and $\left(\left(C_{2}\right)^{2}+\left(C_{0}\right)^{2}\right)^{1 / 2}$.

Proof of Theorem 2(i). Let $\varepsilon>0$ and $\psi=\psi_{0}+\varepsilon \psi_{1}$, where

$$
\widehat{\psi}_{0}=\chi_{[-\pi / 2,-\pi / 4] \cup[\pi / 4, \pi / 2]}, \quad \widehat{\psi}_{1}=\chi_{[-\pi,-\pi / 2] \cup[\pi / 2,3 \pi / 2]} .
$$

Clearly, $\psi_{0}$ is a tight frame wavelet, which is a dilated version of the usual orthonormal Shannon wavelet $\check{\chi}_{[-2 \pi,-\pi] \cup[\pi, 2 \pi]}$. The function $\psi_{1}$ should be regarded as a perturbation term of $\psi=\psi_{0}+\varepsilon \psi_{1}$. The exact form of $\psi_{1}$ is not relevant. In fact, it suffices to take any $\psi_{1} \in L^{2}(\mathbb{R})$ such that $\widehat{\psi}_{1} \in L^{\infty}(\mathbb{R})$ and

$$
\operatorname{supp} \widehat{\psi}_{1} \subset[-\pi,-\pi / 2] \cup[\pi / 2,3 \pi / 2], \quad \widehat{\psi}_{1}(\xi-2 \pi) \widehat{\psi}_{1}(\xi) \not \equiv 0 .
$$

Define the usual sequence of spaces $W_{j}(\psi), j \in \mathbb{Z}$, as

$$
W_{j}(\psi)=\overline{\operatorname{span}}\left\{D^{j} T_{l} \psi: l \in \mathbb{Z}\right\} .
$$


A simple calculation shows that

$$
\begin{aligned}
W_{0}(\psi)=\left\{f \in L^{2}(\mathbb{R}):\right. & \widehat{f}(\xi)=m(\xi) \widehat{\psi}(\xi) \text { for some } 2 \pi \text {-periodic } m(\xi)\} \\
=\left\{f \in L^{2}(\mathbb{R}):\right. & \operatorname{supp} \widehat{f} \subset[-\pi,-\pi / 4] \cup[\pi / 4,3 \pi / 2], \\
& \widehat{f}(\xi-2 \pi)=\widehat{f}(\xi) \text { for all } \xi \in[\pi, 3 \pi / 2]\} .
\end{aligned}
$$

Thus, by scaling we have

$$
\begin{aligned}
W_{j}(\psi)=\left\{f \in L^{2}(\mathbb{R}):\right. & \operatorname{supp} \widehat{f} \subset\left[-2^{j} \pi,-2^{j-2} \pi\right] \cup\left[2^{j-2} \pi, 2^{j-1} 3 \pi\right], \\
& \left.\widehat{f}\left(\xi-2^{j} 2 \pi\right)=\widehat{f}(\xi) \text { for a.e. } \xi \in\left[2^{j} \pi, 2^{j-1} 3 \pi\right]\right\} .
\end{aligned}
$$

In particular,

$$
\check{L}^{2}\left(\left[-2^{j-1} \pi,-2^{j-2} \pi\right] \cup\left[2^{j-2} \pi, 2^{j} \pi\right]\right) \subset W_{j}(\psi) .
$$

Therefore,

$$
V_{0}(\psi)=\overline{\operatorname{span}}\left(\bigcup_{j<0} W_{j}(\psi)\right)
$$

yields

$$
\check{L}^{2}(-\pi / 4, \pi / 2) \subset V_{0}(\psi)
$$

On the other hand,

$$
W_{j}(\psi) \subset \check{L}^{2}(-\pi / 4, \pi / 2) \quad \text { for } j \leq-2 .
$$

Therefore,

$$
\begin{aligned}
V_{0}(\psi)=\left\{f \in L^{2}(\mathbb{R}):\right. & \operatorname{supp} \widehat{f} \subset[-\pi / 2,3 \pi / 4], \\
& \widehat{f}(\xi-\pi)=\widehat{f}(\xi) \text { for a.e. } \xi \in[\pi / 2,3 \pi / 4]\} .
\end{aligned}
$$

Thus, $V_{0}(\psi)$ is not SI. This follows immediately from a basic fact stating that any SI space $V \subset \check{L}^{2}(-\pi, \pi)$ must be of the form $V=\check{L}^{2}(K)$ for some measurable $K \subset(-\pi, \pi)$.

By Theorem $4,\left\{D^{n} T_{l} \psi_{1}\right\}$ is a Bessel sequence. Therefore, by Lemma 1 , $\psi=\psi_{0}+\varepsilon \psi_{1}$ is a frame wavelet for sufficiently small $\varepsilon>0$, since the Bessel constant of $\left\{D^{n} T_{l}\left(\varepsilon \psi_{1}\right)\right\}$ is equal to the product of $\varepsilon^{2}$ and the Bessel constant of $\left\{D^{n} T_{l} \psi_{1}\right\}$.

It is easy to check that $\psi$ and $\psi_{0}$ satisfy the following characterization equations:

$$
\begin{aligned}
\sum_{j \in \mathbb{Z}} \widehat{\psi}\left(2^{j} \xi\right) \overline{\widehat{\psi}_{0}\left(2^{j} \xi\right)} & =1 \quad \text { for a.e. } \xi \\
\sum_{j=0}^{\infty} \widehat{\psi}\left(2^{j} \xi\right) \overline{\widehat{\psi}_{0}\left(2^{j}(\xi+2 \pi q)\right)} & =0 \quad \text { for a.e. } \xi \text { and for } q \text { odd }
\end{aligned}
$$

Therefore, $\psi_{0}$ is an alternate dual wavelet frame to $\psi$, which completes the proof of Theorem 2(i). 
Proof of Theorem 2(ii). Let $1<a<\sqrt{2}$ and let $\psi \in L^{2}(\mathbb{R})$ be given by

$$
\widehat{\psi}=\chi_{[-2 \pi,-\pi] \cup[\pi, 2 \pi]}+a \chi_{[-\pi,-\pi / 2] \cup[\pi / 2, \pi]} .
$$

We claim that $V_{0}(\psi)$ is shift invariant and furthermore

$$
V_{0}(\psi)=\check{L}^{2}(-\pi, \pi)
$$

Since the inclusion " $\subset$ " in (3) is trivial, it remains to show " $\supset$ ".

Take any $g \in \check{L}^{2}(-\pi, \pi)$ with $\|\widehat{g}\|_{\infty} \leq M$ for some constant $M<\infty$. By a simple density argument it suffices to show that $g \in V_{0}(\psi)$. We claim that any such $g$ can be decomposed as

$$
g=\sum_{j=-\infty}^{-1} f_{j}, \quad \text { where } f_{j} \in W_{j}(\psi),
$$

the convergence being in $L^{2}(\mathbb{R})$. We recall that for any $j \in \mathbb{Z}$,

$$
\begin{aligned}
W_{j}(\psi) & =\overline{\operatorname{span}}\left\{D^{j} T_{l} \psi: l \in \mathbb{Z}\right\} \\
& =\left\{f \in L^{2}(\mathbb{R}): \widehat{f}(\xi)=m(\xi) \widehat{\psi}\left(2^{-j} \xi\right) \text { for some } 2^{j+1} \pi \text {-periodic } m(\xi)\right\} .
\end{aligned}
$$

Therefore, each $f_{j} \in W_{j}(\psi)$ is given as

$$
\widehat{f}_{j}(\xi)=m_{j}(\xi) \widehat{\psi}\left(2^{-j} \xi\right) \quad \text { for a.e. } \xi,
$$

for some $2^{j+1} \pi$-periodic $m_{j}(\xi)$.

By induction we define the sequence $\left\{m_{j}\right\}_{j \leq-1}$ and, consequently, $\left\{f_{j}\right\}_{j \leq-1}$ in (4) using (5). Let $K=(-2 \pi,-\pi) \cup(\pi, 2 \pi)$ be the Shannon wavelet set. Define $m_{-1}(\xi)$ by

$$
m_{-1}(\xi)=\widehat{g}(\xi) \quad \text { for } \xi \in 2^{-1} K,
$$

and extend $m_{-1}(\xi)$ to a $\pi$-periodic function on $\mathbb{R}$. Given $m_{-1}(\xi), \ldots, m_{-n}(\xi)$ satisfying the induction hypothesis

$$
\widehat{g}(\xi)=\sum_{j=-n}^{-1} m_{j}(\xi) \widehat{\psi}\left(2^{-j} \xi\right) \quad \text { for a.e. } \xi \in \bigcup_{j=-n}^{-1} 2^{j} K,
$$

we define $m_{-n-1}(\xi)$ by

$$
m_{-n-1}(\xi)=\widehat{g}(\xi)-\sum_{j=-n}^{-1} m_{j}(\xi) \widehat{\psi}\left(2^{-j} \xi\right) \quad \text { for } \xi \in 2^{-n-1} K,
$$

and extend $m_{-n-1}(\xi)$ to a $2^{-n} \pi$-periodic function on $\mathbb{R}$. Since supp $\widehat{\psi} \subset$ $(-2 \pi, 2 \pi)$, it is immediate that

$$
\widehat{g}(\xi)=\sum_{j=-n-1}^{-1} m_{j}(\xi) \widehat{\psi}\left(2^{-j} \xi\right)
$$


for $\xi \in \bigcup_{j=-n}^{-1} 2^{j} K$. Moreover, since $\widehat{\psi}\left(2^{n+1} \xi\right)=1$ for all $\xi \in 2^{-n-1} K$, the equality (7) also holds for $\xi \in 2^{-n-1} K$. This shows the induction hypothesis (6) for $n+1$.

Furthermore, for $\xi \in 2^{-n-1} K$ we have

$$
\left|m_{-n-1}(\xi)\right| \leq|\widehat{g}(\xi)|+\sum_{j=-n}^{-1}\left|m_{j}(\xi)\right|\left|\widehat{\psi}\left(2^{-j} \xi\right)\right| \leq M+\left|m_{-n}(\xi)\right| a,
$$

since $\widehat{\psi}(\xi)=0$ for $\xi \in(-\pi / 2, \pi / 2)$. Therefore,

$$
\left\|m_{-n-1}\right\|_{\infty} \leq M+a\left\|m_{-n}\right\|_{\infty}
$$

and consequently

$$
\left\|m_{-n-1}\right\|_{\infty} \leq M+a M+\ldots+a^{n} M=M \frac{a^{n+1}-1}{a-1} .
$$

By (5),

$$
\left\|f_{j}\right\|_{2}^{2}=\frac{1}{2 \pi}\left\|\widehat{f}_{j}\right\|_{2}^{2} \leq \frac{\left\|m_{j}\right\|_{\infty}^{2}}{2 \pi} \int_{\mathbb{R}}\left|\widehat{\psi}\left(2^{-j} \xi\right)\right|^{2} d \xi \leq \frac{M^{2} a^{-2 j} 2^{j}}{2 \pi(a-1)^{2}}\|\widehat{\psi}\|_{2}^{2} .
$$

Therefore,

$$
\sum_{j=-\infty}^{-1}\left\|f_{j}\right\|_{2} \leq \frac{M\|\psi\|_{2}}{a-1} \sum_{j=-\infty}^{-1}\left(2 / a^{2}\right)^{j}<\infty
$$

By (5) and (6),

$$
\widehat{g}(\xi)=\sum_{j=-\infty}^{-1} \widehat{f}_{j}(\xi) \quad \text { for a.e. } \xi
$$

which shows (4). This completes the proof of (3).

It remains to show that $\psi$ is a frame wavelet which has no affine duals. Indeed, we can represent $\psi=\psi_{0}+a \psi_{1}$, where $\widehat{\psi}_{0}=\chi_{[-2 \pi,-\pi] \cup[\pi, 2 \pi]}$ and $\widehat{\psi}_{1}=\chi_{[-\pi,-\pi / 2] \cup[\pi / 2, \pi]}$. Clearly, $\psi_{0}$ is an orthonormal Shannon wavelet and $\psi_{1}$ is a tight frame wavelet with constant 1 . Therefore, by Lemma $1, \psi=$ $\psi_{0}+a \psi_{1}$ is a frame wavelet since $a>1$.

Finally, we need to show that $\psi$ has no affine duals. On the contrary, suppose that $\phi \in L^{2}(\mathbb{R})$ is an affine dual of $\psi$. Then, by the characterization equations,

$$
\begin{aligned}
& \sum_{j \in \mathbb{Z}} \widehat{\psi}\left(2^{j} \xi\right) \overline{\widehat{\phi}\left(2^{j} \xi\right)}=1 \quad \text { for a.e. } \xi \\
& \sum_{j=0}^{\infty} \widehat{\psi}\left(2^{j} \xi\right) \overline{\widehat{\phi}\left(2^{j}(\xi+2 \pi q)\right)}=0 \quad \text { for a.e. } \xi \text { and for } q \text { odd }
\end{aligned}
$$


Hence, for a.e. $\xi$ with $\pi<|\xi|<2 \pi$, by (9) we have $\widehat{\phi}(\xi+2 \pi q)=0$ for every odd $q$. Thus,

$$
\operatorname{supp} \widehat{\phi} \subset \bigcup_{k \in \mathbb{Z}}(\pi+4 \pi k, 3 \pi+4 \pi k) .
$$

Combining (2), (8), and (10) yields

$$
\widehat{\phi}(\xi)=1 \quad \text { for a.e. } \pi<|\xi|<2 \pi \text {. }
$$

Applying (9) for $-\pi<\xi<-\pi / 2$ and $q=1$ and using (2) and (11) yields

$$
0=\sum_{j=0}^{1} \widehat{\psi}\left(2^{j} \xi\right) \overline{\widehat{\phi}\left(2^{j}(\xi+2 \pi q)\right)}=a+\overline{\widehat{\phi}(2(\xi+2 \pi q))},
$$

which implies

$$
\widehat{\phi}(\xi)=-a \quad \text { for a.e. } 2 \pi<|\xi|<3 \pi .
$$

More generally, define recursively the sequence $\left\{a_{j}\right\}_{j \geq 1}$ by setting $a_{1}=1$ and

$$
a_{j+1}= \begin{cases}2 a_{j} & \text { for odd } j, \\ 2 a_{j}+1 & \text { for even } j .\end{cases}
$$

Note that $a_{j}$ is odd for odd $j$ and $a_{j}$ is even for even $j$. We will show inductively that for all $j \geq 1$,

$$
\widehat{\phi}(\xi)=(-1)^{j-1} a^{j-1} \quad \text { for a.e. } \xi \in\left(a_{j} \pi,\left(a_{j}+1\right) \pi\right) .
$$

Clearly, (13) holds for $j=1,2$ by (11) and (12).

Suppose that (13) holds for even $j$. Applying (9) for $\pi / 2<\xi<\pi$ and $q=a_{j-1}$ and using (2) and (13) yields

$$
0=\sum_{l=0}^{1} \widehat{\psi}\left(2^{l} \xi\right) \overline{\widehat{\phi}\left(2^{l}(\xi+2 \pi q)\right)}=a(-1)^{j-1} a^{j-1}+\overline{\widehat{\phi}(2(\xi+2 \pi q))},
$$

which implies

$$
\widehat{\phi}(\xi)=(-1)^{j} a^{j} \quad \text { for a.e. } \xi \in\left(\left(2 a_{j}+1\right) \pi,\left(2 a_{j}+2\right) \pi\right),
$$

showing (13) for $j+1$.

Likewise, suppose that (13) holds for odd $j$. Applying (9) for $-\pi<\xi<$ $-\pi / 2$ and $q=a_{j-1}+1$ and using (2) and (13) yields

$$
0=\sum_{l=0}^{1} \widehat{\psi}\left(2^{l} \xi\right) \overline{\widehat{\phi}\left(2^{l}(\xi+2 \pi q)\right)}=a(-1)^{j-1} a^{j-1}+\overline{\widehat{\phi}(2(\xi+2 \pi q))},
$$

which implies

$$
\widehat{\phi}(\xi)=(-1)^{j} a^{j} \quad \text { for a.e. } \xi \in\left(2 a_{j} \pi,\left(2 a_{j}+1\right) \pi\right),
$$

showing (13) for $j+1$. 
This completes the proof of (13) for all $j \geq 1$. Since $a \geq 1$, we have $\|\widehat{\phi}\|_{2}=\infty$, which contradicts $\phi \in L^{2}(\mathbb{R})$. This shows that $\psi$ has no affine duals and completes the proof of Theorem 2(ii).

As illustrated above, in contrast to the Riesz basis case, the shift invariance of $V_{0}(\Psi)$ does not reveal all of the information desired about the dual structure of $\Psi$. However, under a special condition, the shift invariance of $V_{0}(\Psi)$ does give more information (see also Theorem 9 ).

TheOREM 5. Suppose $\Psi$ is a frame wavelet which is similar to a semiorthogonal frame wavelet $\Phi$. Then $V_{0}(\Psi)$ is shift invariant if and only if the standard dual of the affine frame generated by $\Psi$ is an affine frame with the same number of generators.

Recall that the frame wavelet $\Phi=\left\{\phi_{1}, \ldots, \phi_{r}\right\}$ is said to be semiorthogonal if $n \neq k$ implies that $\left\langle D^{n} T_{z} \phi_{i}, D^{k} T_{z^{\prime}} \phi_{j}\right\rangle=0$ for all $z, z^{\prime} \in \mathbb{Z}^{d}$ and $i, j=1, \ldots, r$. Frame wavelets $\Psi$ and $\Phi$ are similar if there is an invertible operator $V$ such that

$$
V D^{n} T_{z} \phi_{i}=D^{n} T_{z} \psi_{i} \quad \text { for } n \in \mathbb{Z}, z \in \mathbb{Z}^{d}, i=1, \ldots, r,
$$

i.e., $V$ maps $\Phi$ onto $\Psi$ and $V$ is in the local commutant of the affine system at $\Phi$. Note that $V$ commutes with $D$.

Proof. Clearly, the "if" implication is true. We will show that the "only if" implication is true using the idea of the local commutant [14].

Let $S_{\Phi}$ and $S_{\Psi}$ be the frame operators of $\Phi$ and $\Psi$, respectively. Since the frame wavelet $\Phi$ is semi-orthogonal, it is not hard to show that $S_{\Phi}$ maps

$$
W_{0}(\Phi)=\overline{\operatorname{span}}\left\{T_{z} \phi_{i}: z \in \mathbb{Z}^{d}, i=1, \ldots, r\right\}
$$

onto itself and

$$
S_{\Phi} T_{z} f=T_{z} S_{\Phi} f \quad \text { for all } f \in W_{0}(\Phi), z \in \mathbb{Z}^{d} .
$$

Consequently, the standard dual of $\left\{D^{n} T_{z} \phi_{i}\right\}$ is $\left\{D^{n} T_{z} S_{\Phi}^{-1} \phi_{i}\right\}$.

Next, we claim that the standard dual of $\left\{D^{n} T_{z} \psi_{i}\right\}$ is of the form $\left\{V^{*-1} D^{n} T_{z} S_{\Phi}^{-1} \phi_{i}\right\}$. Indeed, by (14),

$$
\begin{aligned}
S_{\Psi} & =\sum_{i=1}^{r} \sum_{n \in \mathbb{Z}} \sum_{z \in \mathbb{Z}^{d}}\left\langle\cdot, D^{n} T_{z} \psi_{i}\right\rangle D^{n} T_{z} \psi_{i} \\
& =\sum_{i=1}^{r} \sum_{n \in \mathbb{Z}} \sum_{z \in \mathbb{Z}^{d}}\left\langle V^{*}, D^{n} T_{z} \phi_{i}\right\rangle V D^{n} T_{z} \phi_{i}=V S_{\Phi} V^{*} .
\end{aligned}
$$

Thus,

$$
S_{\Psi}^{-1} D^{n} T_{z} \psi_{i}=V^{*-1} S_{\Phi}^{-1} V^{-1} D^{n} T_{z} \psi_{i}=V^{*-1} D^{n} T_{z} S_{\Phi}^{-1} \phi_{i}
$$


Therefore, to show that the standard dual of $\left\{D^{n} T_{z} \psi_{i}\right\}$ is affine with the same number of generators, we must show that

$$
V^{*-1} T_{z} S_{\Phi}^{-1} \phi_{i}=T_{z} V^{*-1} S_{\Phi}^{-1} \phi_{i} \quad \text { for } z \in \mathbb{Z}^{d}, i=1, \ldots, r,
$$

since $V$ commutes with $D$. Since $S_{\Phi}$ maps $W_{0}(\Phi)$ isomorphically onto itself, it suffices to show that

$$
V^{*-1} T_{m} \phi_{i}=T_{m} V^{*-1} \phi_{i} \quad \text { for } m \in \mathbb{Z}^{d}, i=1, \ldots, r .
$$

For $n>0$, we have

$$
\left\langle V^{*-1} T_{m} \phi_{i}, D^{n} T_{z} \psi_{j}\right\rangle=\left\langle\phi_{i}, T_{-m} V^{-1} D^{n} T_{z} \psi_{j}\right\rangle=\left\langle\phi_{i}, T_{-m} D^{n} T_{z} \phi_{j}\right\rangle=0 .
$$

Likewise,

$$
\left\langle T_{m} V^{*-1} \phi_{i}, D^{n} T_{z} \psi_{j}\right\rangle=\left\langle\phi_{i}, V^{-1} T_{-m} D^{n} T_{z} \psi_{j}\right\rangle=\left\langle\phi_{i}, T_{-m} D^{n} T_{z} \phi_{j}\right\rangle=0 .
$$

For $n<0$, we have

$$
\left\langle V^{*-1} T_{m} \phi_{i}, D^{n} T_{z} \psi_{j}\right\rangle=\left\langle\phi_{i}, T_{-m} V^{-1} D^{n} T_{z} \psi_{j}\right\rangle=\left\langle\phi_{i}, T_{-m} D^{n} T_{z} \phi_{j}\right\rangle=0,
$$

since $T_{-m} D^{n} T_{z} \phi_{j} \in V_{0}(\Phi)$. Likewise,

$$
\left\langle T_{m} V^{*-1} \phi_{i}, D^{n} T_{z} \psi_{j}\right\rangle=\left\langle\phi_{i}, V^{-1} T_{-m} D^{n} T_{z} \psi_{j}\right\rangle=\left\langle\phi_{i}, V^{-1} f\right\rangle=0,
$$

where $f \in V_{0}(\Psi)$, since it is shift invariant. Finally, for $n=0$,

$$
\begin{aligned}
\left\langle V^{*-1} T_{m} \phi_{i}, T_{z} \psi_{j}\right\rangle & =\left\langle\phi_{i}, T_{-m} V^{-1} T_{z} \psi_{j}\right\rangle=\left\langle\phi_{i}, V^{-1} T_{-m} T_{z} \psi_{j}\right\rangle \\
& =\left\langle T_{m} V^{*-1} \phi_{i}, T_{z} \psi_{j}\right\rangle .
\end{aligned}
$$

Therefore, (15) holds, which completes the proof of Theorem 5.

Since every Riesz wavelet is similar to an orthonormal wavelet, as an immediate corollary of Theorem 5 we obtain the previously mentioned result for Riesz wavelets [15, 21, 22].

Corollary 2. Suppose $\Psi$ is a Riesz wavelet. Then $V_{0}(\Psi)$ is shift invariant if and only if the (unique) dual of $\left\{D^{n} T_{z} \psi: n \in \mathbb{Z}, z \in \mathbb{Z}^{d}, \psi \in \Psi\right\}$ is of the form $\left\{D^{n} T_{z} \phi: n \in \mathbb{Z}, z \in \mathbb{Z}^{d}, \phi \in \Phi\right\}$, with the cardinality of the sets $\Psi$ and $\Phi$ being equal.

1.1. Quasi-affine frames. We now set out to prove Theorem 3. Let $S_{\Psi}^{q}$ denote the frame transform of the quasi-affine system $\mathcal{U}^{q}(\Psi)$, i.e.

$$
\begin{gathered}
S_{\Psi}^{q}: L^{2}\left(\mathbb{R}^{d}\right) \rightarrow L^{2}\left(\mathbb{R}^{d}\right), \\
f \mapsto \sum_{\psi \in \Psi} \sum_{n \geq 0} \sum_{z \in \mathbb{Z}^{d}}\left\langle f, D^{n} T_{z} \psi\right\rangle D^{n} T_{z} \psi+\sum_{\psi \in \Psi} \sum_{n<0} \sum_{z \in \mathbb{Z}^{d}}\left\langle f, T_{z} \widetilde{D}^{n} \psi\right\rangle T_{z} \widetilde{D}^{n} \psi .
\end{gathered}
$$

Clearly, the standard dual of $\mathcal{U}^{q}(\Psi)$ has the form $\mathcal{U}^{q}(\Phi)$ if and only if $\left(S_{\Psi}^{q}\right)^{-1} \in \mathcal{C}_{\psi}\left(\mathcal{U}^{q}\right)$ for all $\psi \in \Psi$. With the quasi-affine system, this is much easier to determine than with the affine system. 
Proposition 1. Suppose $\mathcal{U}^{q}(\Psi)$ is a complete system (not necessarily a frame) in $L^{2}\left(\mathbb{R}^{d}\right)$. For any operator $S \in \mathcal{B}\left(L^{2}\left(\mathbb{R}^{d}\right)\right), S \in \mathcal{C}_{\psi}\left(\mathcal{U}^{q}\right)$ for all $\psi \in \Psi$ if and only if $S \in\left\{D, T_{z}: z \in \mathbb{Z}^{d}\right\}^{\prime}$, the joint commutant of $D$ and $T_{z}$.

Proof. The "if" implication is obvious. Suppose that $S \in \mathcal{C}_{\psi}\left(\mathcal{U}^{q}\right)$ for all $\psi \in \Psi$. Define the unitary counterpart of the quasi-affine system as follows:

$$
\mathcal{V}^{q}:=\left\{D^{n} T_{z}: n \geq 0, z \in \mathbb{Z}^{d}\right\} \cup\left\{T_{z} D^{n}: n<0, z \in \mathbb{Z}^{d}\right\} .
$$

Note that $\mathcal{V}^{q} \Psi$ is still a complete system, and that $S \in \mathcal{C}_{\psi}\left(\mathcal{U}^{q}\right)$ for all $\psi \in \Psi$ if and only if $S \in \mathcal{C}_{\psi}\left(\mathcal{V}^{q}\right)$ for all $\psi \in \Psi$. For the purposes of this proof, let $C$ denote an arbitrary element of $\mathcal{V}^{q}$. It suffices to show that $S T_{k} C \psi=T_{k} S C \psi$ and $S D C \psi=D S C \psi$ for all $\psi \in \Psi$.

We note that $\mathcal{V}^{q}$ is a shift invariant system, i.e., $T_{k} \mathcal{V}^{q} \subset \mathcal{V}^{q}$ for all $k \in \mathbb{Z}^{d}$. We claim that this is also true for $D^{-1}$. Indeed, for $n \geq 1, D^{-1} D^{n} T_{z} \in \mathcal{V}^{q}$. Now, for $n \leq 0, D^{-1} T_{z} D^{n}=T_{A z} D^{n-1} \in \mathcal{V}^{q}$, since for $f \in L^{2}\left(\mathbb{R}^{d}\right)$,

$$
\begin{aligned}
D^{-1} T_{z} f(x) & =|\operatorname{det} A|^{-1 / 2} f\left(A^{-1} x-z\right)=|\operatorname{det} A|^{-1 / 2} f\left(A^{-1}(x-A z)\right) \\
& =T_{A z} D^{-1} f(x) .
\end{aligned}
$$

Define two mappings on $\mathcal{V}^{q}$ by

$$
\gamma: \mathcal{V}^{q} \rightarrow \mathcal{V}^{q}, \quad C \mapsto T_{k} C ; \quad \eta: \mathcal{V}^{q} \rightarrow \mathcal{V}^{q}, \quad C \mapsto D^{-1} C .
$$

We have

$$
\begin{aligned}
S T_{k} C \psi & =S \gamma(C) \psi=\gamma(C) S \psi=T_{k} C S \psi=T_{k} S C \psi, \\
S D^{-1} C \psi & =S \eta(C) \psi=\eta(C) S \psi=D^{-1} C S \psi=D^{-1} S C \psi .
\end{aligned}
$$

It now follows that $S \in\left\{T_{z}: z \in \mathbb{Z}^{d}\right\}^{\prime}$ and $S \in\{D\}^{\prime}$.

Lemma 2. Let $S$ be a bounded operator on $L^{2}\left(\mathbb{R}^{d}\right)$. Then $S \in\left\{T_{z}\right.$ : $\left.z \in \mathbb{Z}^{d}\right\}^{\prime} \cap\{D\}^{\prime}$ if and only if $S$ is an $A^{*}$-dilation periodic Fourier multiplier, i.e., there exists a function $s(\xi) \in L^{\infty}\left(\mathbb{R}^{d}\right)$ such that

$$
\widehat{S f}(\xi)=s(\xi) \widehat{f}(\xi)
$$

where $s(\xi)=s\left(A^{*} \xi\right)$ for a.e. $\xi$.

Proof. If $S \in\left\{T_{z}: z \in \mathbb{Z}^{d}\right\}^{\prime} \cap\{D\}^{\prime}$, then $S$ commutes with the von Neumann algebra generated by $\left\{T_{z}: z \in \mathbb{Z}^{d}\right\}$ and $D$. Note that this von Neumann algebra contains the operators

$$
T_{A^{j} z}=D^{-j} T_{z} D^{j}
$$

for all $j \in \mathbb{Z}$ and $z \in \mathbb{Z}^{d}$. Therefore, this von Neumann algebra contains all translation operators, i.e. $T_{\alpha}$ for all $\alpha \in \mathbb{R}^{d}$. It now follows that $S$ is a Fourier multiplier. 
To see that $s(\xi)$ is " $A^{*}$-dilation periodic", note that

$$
\begin{aligned}
|\operatorname{det} A|^{-1 / 2} s\left(A^{*-1} \xi\right) \widehat{f}\left(A^{*-1} \xi\right) & =(D S f)^{\wedge}(\xi)=(S D f)^{\wedge}(\xi) \\
& =s(\xi)|\operatorname{det} A|^{-1 / 2} \widehat{f}\left(A^{*-1} \xi\right) .
\end{aligned}
$$

The converse follows immediately.

Proof of Theorem 3. We first introduce some notation and results from [18] (see also [23]). For a frame of the form $\left\{T_{C_{p} k} g_{p}: k \in \mathbb{Z}^{d}, p \in \mathcal{P}\right\}$, where $C_{p} \in \mathrm{GL}(d, \mathbb{R})$ and $\mathcal{P}$ is a countable index set, define the following continuous function:

$$
w(x)=\sum_{p \in \mathcal{P}} \sum_{k \in \mathbb{Z}^{d}}\left\langle T_{x} f, T_{C_{p} k} g_{p}\right\rangle\left\langle T_{C_{p} k} g_{p}, T_{x} f\right\rangle=\left\langle S_{g} T_{x} f, T_{x} f\right\rangle,
$$

where $S_{g}$ is the frame operator of the above frame.

Let $\Lambda=\left\{C_{p}^{*-1} z: z \in \mathbb{Z}^{d}, p \in \mathcal{P}\right\}$, and for $\alpha \in \Lambda$, let $P_{\alpha}=\{p \in \mathcal{P}$ : $\left.C_{p}^{*} \alpha \in \mathbb{Z}^{d}\right\}$. Let

$\mathcal{D}:=\left\{f \in L^{2}\left(\mathbb{R}^{d}\right): \widehat{f} \in L^{\infty}\left(\mathbb{R}^{d}\right)\right.$ and $\widehat{f}$ compactly supported and supported away from 0$\}$.

Note that $\mathcal{D}$ is dense in $L^{2}\left(\mathbb{R}^{d}\right)$. It is shown in [18] that $w(x)$ is in fact an almost periodic function given by

where

$$
w(x)=\sum_{\alpha \in \Lambda} \widehat{w}(\alpha) e^{2 \pi i \alpha \cdot x},
$$

$$
\widehat{w}(\alpha)=\frac{1}{2 \pi} \int_{\mathbb{R}^{d}} \widehat{f}(\xi) \overline{\widehat{f}(\xi+2 \pi \alpha)} \sum_{p \in P_{\alpha}}\left|\operatorname{det} C_{p}\right|^{-1} \overline{\widehat{g}_{p}(\xi)} \widehat{g}_{p}(\xi+2 \pi \alpha) d \xi .
$$

Additionally, it is shown that $w(x)$ is identically constant if and only if $\widehat{w}(\alpha)=0$ for all $\alpha \neq 0$ (see [18, Theorem 2.1 and Proposition 2.4]).

If a quasi-affine system is a frame, then it satisfies the necessary integrability condition mentioned above [18, Proposition 5.6]. Write

$$
\begin{aligned}
\mathcal{U}^{q}(\Psi)= & \left\{T_{A^{-n} z} D^{n} \psi_{i}: z \in \mathbb{Z}^{d}, n \geq 0, i=1, \ldots, r\right\} \\
& \cup\left\{T_{z} \widetilde{D}^{n} \psi_{i}: z \in \mathbb{Z}^{d}, n<0, i=1, \ldots, r\right\} .
\end{aligned}
$$

Thus, $\mathcal{P}=\mathbb{Z} \times\{1, \ldots, r\}$,

$$
C_{(n, i)}=\left\{\begin{array}{ll}
A^{-n} & \text { for } n \geq 0, \\
I & \text { for } n<0,
\end{array} \quad g_{(n, i)}= \begin{cases}D^{n} \psi_{i} & \text { for } n \geq 0 \\
\widetilde{D}^{n} \psi_{i} & \text { for } n<0\end{cases}\right.
$$

For the remainder of the proof, we shall suppress the index $i$. Since $A^{*}$ is integer-valued, $\Lambda=\mathbb{Z}^{d}$. Thus, let $\alpha \in \mathbb{Z}^{d}$ and write $\alpha=A^{* n} q$, where $n \geq 0$ and $q \in \mathbb{Z}^{d} \backslash A^{*} \mathbb{Z}^{d}$. We have

$$
\begin{aligned}
\mathcal{P}_{\alpha} & =\left\{p \in \mathbb{Z}: C_{p}^{*} A^{* n} q \in \mathbb{Z}^{d}\right\}=\{p \in \mathbb{Z}: p<0\} \cup\left\{p \geq 0: A^{*-p} A^{* n} q \in \mathbb{Z}^{d}\right\} \\
& =\{p \in \mathbb{Z}: p \leq n\} .
\end{aligned}
$$


Therefore,

$$
\begin{aligned}
& \text { (17) } \quad \sum_{p \in \mathcal{P}_{\alpha}}\left|\operatorname{det} C_{p}\right|^{-1} \overline{\hat{g}_{p}(\xi)} \widehat{g}_{p}(\xi+2 \pi \alpha) \\
& =\sum_{\psi \in \Psi} \sum_{p<0} \widehat{\widehat{\psi}\left(A^{*-p} \xi\right)} \widehat{\psi}\left(A^{*-p}\left(\xi+2 \pi A^{* n} q\right)\right) \\
& \quad+\sum_{\psi \in \Psi} \sum_{p=0}^{n}\left|\operatorname{det} A^{p}\right| \sqrt{\left|\operatorname{det} A^{-p}\right|} \widehat{\widehat{\psi}\left(A^{*-p} \xi\right)} \sqrt{\left|\operatorname{det} A^{-p}\right|} \widehat{\psi}\left(A^{*-p}\left(\xi+2 \pi A^{* n} q\right)\right) \\
& =\sum_{\psi \in \Psi} \sum_{j=-n}^{\infty} \widehat{\widehat{\psi}\left(A^{* j} \xi\right)} \widehat{\psi}\left(A^{* j}\left(\xi+2 \pi A^{* n} q\right)\right) \\
& =\sum_{\psi \in \Psi} \sum_{j=0}^{\infty} \overline{\widehat{\psi}\left(A^{* j} A^{*-n} \xi\right)} \widehat{\psi}\left(A^{* j}\left(A^{*-n} \xi+2 \pi q\right)\right)
\end{aligned}
$$

Suppose that the canonical dual of $\mathcal{U}^{q}(\Psi)$ is of the form $\mathcal{U}^{q}(\Phi)$, whence by Proposition $1, S_{\Psi}^{q} \in\left\{D, T_{z}\right\}^{\prime}$. For $f \in \mathcal{D}$, the almost periodic function $w(x)$, as defined above, is constant, since

$$
w(x)=\left\langle T_{x} S_{\Psi}^{q} f, T_{x} f\right\rangle=\left\langle S_{\Psi}^{q} f, f\right\rangle .
$$

Thus, (1) holds for $q \in \mathbb{Z}^{d} \backslash A^{*} \mathbb{Z}^{d}$ by (16) and (17).

Conversely, suppose (1) holds for $q \in \mathbb{Z}^{d} \backslash A^{*} \mathbb{Z}^{d}$. Then the almost periodic function $w(x)$ is constant, whence $S_{\Psi}^{q} T_{x} f=T_{x} S_{\Psi}^{q} f$ for all $f \in \mathcal{D}$. Therefore, $S_{\Psi}^{q}$ is a Fourier multiplier. We claim that the symbol for $S_{\Psi}^{q}$ is

$$
s(\xi)=\sum_{\psi \in \Psi} \sum_{j \in \mathbb{Z}} \widehat{\psi}\left(A^{* j} \xi\right) \overline{\widehat{\psi}\left(A^{* j} \xi\right)} .
$$

This can be seen by evaluating $w(x)$ at $x=0$ :

$$
w(0)=\left\langle S_{\Psi}^{q} f, f\right\rangle=\frac{1}{2 \pi} \int_{\mathbb{R}^{d}} \widehat{f}(\xi) \overline{\hat{f}(\xi)} \sum_{\psi \in \Psi} \sum_{j \in \mathbb{Z}} \widehat{\psi}\left(A^{* j} \xi\right) \overline{\widehat{\psi}\left(A^{* j} \xi\right)} d \xi .
$$

Clearly, $s\left(A^{*} \xi\right)=s(\xi)$, whence by Lemma $2, S_{\Psi}^{q} \in\left\{D, T_{z}\right\}^{\prime}$, and the standard dual of $\mathcal{U}^{q}(\Psi)$ is of the form $\mathcal{U}^{q}(\Phi)$.

It is also possible to give an alternative proof of Theorem 3, which is based on the theory of shift invariant spaces and more precisely on techniques of dual Gramians introduced by Ron and Shen [24]. We will employ the following result, which can be found in [4, Theorem 2.5(ii)] or [24].

TheOREM 6. Suppose $\mathcal{A}$ is a countable collection of functions in $L^{2}\left(\mathbb{R}^{d}\right)$. For a.e. $\xi \in[-\pi, \pi)^{d}$, let $\widetilde{G}(\xi)$ be the dual Gramian of $\mathcal{A}$ at $\xi$, i.e., $\widetilde{G}(\xi)$ is the frame operator of

$$
\left\{(\widehat{\varphi}(\xi+2 \pi k))_{k \in \mathbb{Z}^{d}}: \varphi \in \mathcal{A}\right\} \subset \ell^{2}\left(\mathbb{Z}^{d}\right) .
$$


Then the shift invariant system

$$
E(\mathcal{A}):=\left\{T_{z} \varphi: \varphi \in \mathcal{A}, z \in \mathbb{Z}^{d}\right\}
$$

is a frame for $L^{2}\left(\mathbb{R}^{d}\right)$ with constants $C_{1}$ and $C_{2}$ if and only if $\widetilde{G}(\xi)$ is bounded for a.e. $\xi$ and

$$
C_{1}\|v\|^{2} \leq\langle\widetilde{G}(\xi) v, v\rangle \leq C_{2}\|v\|^{2} \quad \text { for all } v \in \ell^{2}\left(\mathbb{Z}^{d}\right) \text { and a.e. } \xi \in[-\pi, \pi)^{d},
$$

i.e., the spectrum $\sigma(\widetilde{G}(\xi))$ lies in $\left[C_{1}, C_{2}\right]$ for a.e. $\xi$.

We will also need an additional result tying a frame operator of a shift invariant system to its corresponding dual Gramians using range operators; see [4, Theorem 5.1]. For a definition and properties of range operators we refer to [4, Section 4].

THEOREM 7. Suppose $\mathcal{A}$ is a countable collection of functions in $L^{2}\left(\mathbb{R}^{d}\right)$ such that the shift invariant system $E(\Phi)$ is a frame for $L^{2}\left(\mathbb{R}^{d}\right)$. Let $S$ be the frame operator corresponding to this system. Then the dual Gramian $\widetilde{G}(\xi)$ is the range operator corresponding to $S$, that is,

$$
(\widehat{S f}(\xi+2 \pi k))_{k \in \mathbb{Z}^{d}}=\widetilde{G}(\xi)\left((\widehat{f}(\xi+2 \pi k))_{k \in \mathbb{Z}^{d}}\right)
$$

$$
\text { for a.e. } \xi \text { and } f \in L^{2}\left(\mathbb{R}^{d}\right) \text {. }
$$

In particular, $S$ is a Fourier multiplier if and only if $\widetilde{G}(\xi)$ is a diagonal operator on $\ell^{2}\left(\mathbb{Z}^{d}\right)$ for a.e. $\xi$.

Another proof of Theorem 3. Since $\mathcal{U}^{q}(\Psi)$ is a shift invariant system, it can be written as $E(\mathcal{A})$ for some countable collection $\mathcal{A} \subset L^{2}\left(\mathbb{R}^{d}\right)$. By $[5$, Lemma 2.3], its dual Gramian $\widetilde{G}(\xi)$ is equal to

$$
\begin{aligned}
\left\langle\widetilde{G}(\xi) \mathbf{e}_{k}, \mathbf{e}_{k}\right\rangle & =\sum_{\psi \in \Psi} \sum_{j \in \mathbb{Z}}\left|\widehat{\psi}\left(A^{* j}(\xi+2 \pi k)\right)\right|^{2} & & \text { for } k \in \mathbb{Z}^{d}, \\
\left\langle\widetilde{G}(\xi) \mathbf{e}_{k}, \mathbf{e}_{l}\right\rangle & =t_{q}\left(A^{*-n}(\xi+2 \pi k)\right) & & \text { for } k \neq l \in \mathbb{Z}^{d},
\end{aligned}
$$

where $q \in \mathbb{Z}^{d} \backslash A^{*} \mathbb{Z}^{d}$ and $n \geq 0$ are unique elements satisfying $l-k=A^{* n} q$, and $\left\{\mathbf{e}_{k}\right\}_{k \in \mathbb{Z}^{d}}$ is the standard orthonormal basis of $\ell^{2}\left(\mathbb{Z}^{d}\right)$.

Suppose that the canonical dual of $\mathcal{U}^{q}(\Psi)$ is of the form $\mathcal{U}^{q}(\Phi)$. Then by Proposition 1 and Lemma 2, the frame operator $S_{\Psi}^{q}$ is a Fourier multiplier. Therefore, by (21) and Theorem 7, (1) holds.

Conversely, suppose that (1) holds. Then, by (19)-(21), and Theorem 7 , the frame operator $S_{\Psi}^{q}$ is a Fourier multiplier with the symbol $s(\xi)$ given by (18). Since $s(\xi)=s\left(A^{*} \xi\right)$, the canonical dual of $\mathcal{U}^{q}(\Psi)$ is of the form $\mathcal{U}^{q}(\Phi)$ by Proposition 1 and Lemma 2. This completes the proof of Theorem 3.

Ron and Shen showed in [25, Theorem 1(c)] that if the canonical dual of $\mathcal{U}^{q}(\Psi)$ is of the form $\mathcal{U}^{q}(\Phi)$, then $\mathcal{U}(\Phi)$ is the canonical dual of $\mathcal{U}(\Psi)$. As seen from Theorem 3 and from Examples 1 and 2 in Section 3, the converse is not true. 
COROLlary 3. There exist frame wavelets $\mathcal{U}(\Psi)$ with canonical dual of the form $\mathcal{U}(\Phi)$, but the canonical dual of $\mathcal{U}^{q}(\Psi)$ is not of the form $\mathcal{U}^{q}(\Phi)$.

REMARK 1. We note that in Corollary $3, \mathcal{U}^{q}(\Phi)$ is still an alternate dual of $\mathcal{U}^{q}(\Psi)$. Indeed, Ron and Shen [24] showed that $\mathcal{U}(\Psi)$ is a frame if and only if $\mathcal{U}^{q}(\Psi)$ is a frame and the frame bounds are preserved. This was shown in [24] under some decay conditions on $\Psi$ and $\Phi$, which were subsequently removed in [12]. Furthermore, $\mathcal{U}(\Phi)$ is a dual of $\mathcal{U}(\Psi)$ if and only if $\mathcal{U}^{q}(\Phi)$ is a dual of $\mathcal{U}^{q}(\Psi)$ (see [3, Theorem 4.1]).

Another consequence of Proposition 1 is that similarity of quasi-affine frames preserves nice properties, unlike similarity in the affine case.

TheOREM 8. Suppose $\mathcal{U}^{q}(\Psi)$ and $\mathcal{U}^{q}(\Phi)$ are similar. Then the following hold:

(i) if the standard dual of $\Psi$ has the quasi-affine structure, then so does the standard dual of $\Phi$;

(ii) if $V_{0}(\Psi)$ is shift invariant, then so is $V_{0}(\Phi)$, and moreover, $\Psi$ and $\Phi$ are core equivalent.

Proof. Recall that $\mathcal{U}^{q}(\Psi)$ and $\mathcal{U}^{q}(\Phi)$ are similar if there exists an invertible operator $V$ such that

$$
V C \psi_{i}=C \phi_{i} \quad \text { for all } C \in \mathcal{U}^{q}, i=1, \ldots, r .
$$

This is equivalent to saying that $V \in \mathcal{C}_{\psi}\left(\mathcal{U}^{q}\right)$ for all $\psi \in \Psi$ and $V$ maps $\Psi$ to $\Phi$. By Proposition $1, V \in\left\{D, T_{z}: z \in \mathbb{Z}^{d}\right\}^{\prime}$.

Let $S_{\Psi}^{q}$ and $S_{\Phi}^{q}$ denote the frame operators of $\mathcal{U}^{q}(\Psi)$ and $\mathcal{U}^{q}(\Phi)$, respectively. By the hypothesis of (i), $S_{\Psi}^{q} \in\left\{D, T_{z}: z \in \mathbb{Z}^{d}\right\}^{\prime}$. We have

$$
\begin{aligned}
S_{\Phi}^{q} & =\sum_{\phi \in \Phi} \sum_{C \in \mathcal{U}^{q}}\langle\cdot, C \phi\rangle C \phi=\sum_{\psi \in \Psi} \sum_{C \in \mathcal{U}^{q}}\langle\cdot, V C \psi\rangle V C \psi \\
& =V\left(\sum_{\psi \in \Psi} \sum_{C \in \mathcal{U}^{q}}\left\langle V^{*} \cdot, C \psi\right\rangle C \psi\right)=V S_{\Psi}^{q} V^{*} .
\end{aligned}
$$

Therefore, $S_{\Phi}^{q} \in\left\{D, T_{z}: z \in \mathbb{Z}^{d}\right\}^{\prime}$, which shows (i) by Proposition 1 .

To show (ii), note that by Proposition $1, V \in \mathcal{C}_{\psi}(\mathcal{U})$, so $V$ maps $D^{n} T_{z} \psi_{i}$ to $D^{n} T_{z} \phi_{i}$ for all $n \in \mathbb{Z}, z \in \mathbb{Z}^{d}$, and $i=1, \ldots, r$. Thus, $V$ maps $V_{0}(\Psi)$ to $V_{0}(\Phi)$; by definition, $V$ is one-to-one. Furthermore, $V$ has dense range. Finally, $V \in\left\{T_{z}: z \in \mathbb{Z}^{d}\right\}^{\prime}$; whence, if $V_{0}(\Psi)$ is shift invariant, then so is $V_{0}(\Phi)$. Moreover, taking the partial isometry part of the polar decomposition of $V$ yields the necessary intertwining operator.

REMARK 2. We remark that Theorem 8 is not true for the affine case; let $\psi$ be an orthonormal wavelet and $\phi$ be a Riesz wavelet that is not biorthogonal. Then clearly $\mathcal{U}(\psi)$ and $\mathcal{U}(\phi)$ are similar, but neither item (i) nor (ii) above holds. 


\section{Applications of Theorem 1}

2.1. Oversampling affine frames. In this subsection we consider several applications of Theorem 1 regarding oversampling of an affine frame. The classical oversampling result is the Chui-Shi second oversampling theorem which says that if

$$
\left\{2^{n / 2} \psi\left(2^{n} \cdot-l\right): n, l \in \mathbb{Z}\right\}
$$

is a tight frame and $N$ is odd, then

$$
\left\{2^{n / 2} N^{-1 / 2} \psi\left(2^{n} \cdot-l / N\right): n, l \in \mathbb{Z}\right\}
$$

is also tight with the same frame bound [11]. It is known that if $N$ is even, tightness can be lost, and exact conditions for when oversampling by even $N$ preserves tightness are given in [8]. More general results can be found in [10].

Here we are interested in the case when the oversampling is accomplished by the dilation matrix, i.e. we consider the frame given by $\left\{D^{n} T_{z} D^{-1} \psi\right\}$.

Lemma 3. Let $\Psi=\left\{\psi_{1}, \ldots, \psi_{r}\right\}$ be a frame wavelet such that $V_{0}(\Psi)$ is shift invariant, and define $\Phi=\left\{\phi_{1}, \ldots, \phi_{r}\right\}:=\left\{D^{-1} \psi_{1}, \ldots, D^{-1} \psi_{r}\right\}$. Then $V_{0}(\Phi)=V_{-1}(\Psi)$.

Proof. We write out both sides of the equality:

$$
\begin{aligned}
V_{0}(\Phi) & =\overline{\operatorname{span}}\left\{D^{n} T_{z} D^{-1} \psi_{i}: n<0, i=1, \ldots, r\right\} \\
& =\overline{\operatorname{span}}\left\{D^{n-1} T_{A^{-1} z} \psi_{i}: n<0, i=1, \ldots, r\right\} \\
& =\overline{\operatorname{span}}\left\{D^{n} T_{A^{-1} z} \psi_{i}: n<-1, i=1, \ldots, r\right\}
\end{aligned}
$$

and

$$
V_{-1}(\Psi)=\overline{\operatorname{span}}\left\{D^{n} T_{z} \psi_{i}: n<-1, i=1, \ldots, r\right\} .
$$

We see immediately that since $A^{-1} \mathbb{Z}^{d} \supset \mathbb{Z}^{d}, V_{-1}(\Psi) \subset V_{0}(\Phi)$. However, note that $T_{A^{-1} z} \psi_{i} \in V_{1}(\Psi)$ for all $z \in \mathbb{Z}^{d}$ and $i=1, \ldots, r$. Therefore, if $n \leq-2$, then $D^{n} T_{A^{-1} z} \psi_{i} \in V_{-1}(\Psi)$, whence it follows that $V_{0}(\Phi) \subset V_{-1}(\Psi)$.

If $\Psi$ is semi-orthogonal, then $V_{-1}(\Psi)$ is shift invariant if and only if $W_{0}(\Psi)$ is shift invariant by the lattice $A^{-1} \mathbb{Z}^{d}$, which converts to a support condition of the Fourier transform of $\Psi$ (see [26]). As a corollary, we have the following:

Theorem 9. If $\Psi=\left\{\psi_{1}, \ldots, \psi_{r}\right\}$ is a semi-orthogonal wavelet, then the standard dual of $D^{-1} \Psi$ is an affine frame of the form $\left\{D^{n} T_{z} \phi_{i}: n \in \mathbb{Z}\right.$, $\left.z \in \mathbb{Z}^{d}, i=1, \ldots, r\right\}$ if and only if $V_{0}\left(D^{-1} \Psi\right)$ is shift invariant. In particular, if $\Psi$ is a tight, semi-orthogonal wavelet, then either

(i) $D^{-1} \Psi$ is a tight, semi-orthogonal frame wavelet, or

(ii) the standard dual of $D^{-1} \Psi$ is not an affine frame.

Proof. The "only if" implication follows from Theorem 1. 
Conversely, suppose $V_{0}\left(D^{-1} \Psi\right)$ is shift invariant. Since $\Psi$ is semi-orthogonal, $V_{0}(\Psi)$ is shift invariant, and by Lemma $3, V_{0}\left(D^{-1} \Psi\right)=V_{-1}(\Psi)$. Hence,

$$
W_{-1}(\Psi)=\overline{\operatorname{span}}\left\{D^{-1} T_{z} \psi_{i}: z \in \mathbb{Z}^{d}, i=1, \ldots, r\right\}=V_{0}(\Psi) \ominus V_{-1}(\Psi)
$$

is also shift invariant and $W_{0}\left(D^{-1} \Psi\right)=W_{-1}(\Psi)$. Therefore, $W_{j}\left(D^{-1} \Psi\right) \perp$ $W_{n}\left(D^{-1} \Psi\right)$ for $j \neq n \in \mathbb{Z}$ and consequently $D^{-1} \Psi$ is also a semi-orthogonal frame wavelet, and so its standard dual is affine.

It follows that if $\Psi$ is a tight, semi-orthogonal frame wavelet such that $V_{-1}(\Psi)$ is shift invariant, then $D^{-1} \Psi$ is also a tight, semi-orthogonal frame wavelet.

COROLLARY 4. If $\Psi$ is a compactly supported orthonormal wavelet, then the standard dual of $D^{-1} \Psi$ is not of the form $\left\{D^{n} T_{z} \phi_{i}: n \in \mathbb{Z}, z \in \mathbb{Z}^{d}, i=\right.$ $1, \ldots, r\}$.

REMARK 3. It is interesting to note that it is possible to oversample by $D^{-1}$ a second time and regain tightness. For example, if $\psi$ is a Meyer orthonormal wavelet, then $D^{-1} \psi$ has the property that $V_{0}\left(D^{-1} \psi\right)$ is not shift invariant, and hence its standard dual is not an affine frame. However, it is easy to check that $D^{-2} \psi$ is a tight frame wavelet (see Example 3 ). There we show that $D^{-1} \psi$ has no alternate duals of the form $\left\{D^{n} T_{l} \phi\right\}$ either. Moreover, $D^{-1} \psi$ is MRA based (see [16]) in the sense that $D^{-1} \psi \in V_{1}(\psi)$. So it is possible for a frame wavelet to be MRA based and have no affine frame duals.

2.2. Periods of affine frames. In this subsection we turn our attention to univariate affine frames associated with an integer dilation factor $A,|A| \geq 2$. Daubechies and Han [15] have introduced the notion of the period of a dyadic affine frame (that is, when $A=2$ ), which can be easily transplanted to a non-dyadic situation.

Definition 1. Suppose that $\Psi=\left\{\psi_{1}, \ldots, \psi_{r}\right\} \subset L^{2}(\mathbb{R})$ is a frame wavelet associated with an integer dilation factor $A,|A| \geq 2$. The period of $\Psi$ is the smallest integer $k \geq 1$ such that for all $f \in \overline{\operatorname{span}}\left\{T_{z} \psi: z \in \mathbb{Z}, \psi \in \Psi\right\}$,

$$
T_{k m} S^{-1} f=S^{-1} T_{k m} f \quad \text { for all } m \in \mathbb{Z},
$$

where $S$ is the frame operator of $\Psi$. If there is no such $k$ then we say that the period of $\Psi$ is 0 .

We remark that the period of $\Psi$ can be equivalently defined as the smallest integer $k \geq 1$ such that $S^{-1}$ is in the local commutant of $\left\{D^{n} T_{k m}\right.$ : $n, m \in \mathbb{Z}\}$ of $T_{z} \psi$ for all $z \in \mathbb{Z}$ and $\psi \in \Psi$.

In their paper [15], Daubechies and Han pose the question of whether the period of a dyadic frame wavelet is always either 0 or $2^{J}$ for some whole number $J$. We will show that the answer is positive for Riesz wavelets. The remaining case of non-Riesz affine frames remains open. 
We start by showing the following result which sheds some light on the period of an affine frame.

Proposition 2. If $\Psi$ is a frame wavelet and $V_{0}(\Psi)$ is shift invariant by the lattice $M \mathbb{Z}$ then the period of $\Psi$ divides $M$. If $\Psi$ generates a Riesz basis, then the period of $\Psi$ divides $M$ if and only if $V_{0}(\Psi)$ is shift invariant by the lattice $M \mathbb{Z}$.

Proof. If the period of $\Psi$ divides $M$, then the standard dual of the frame $\left\{D^{n} T_{M l} T_{j} \psi_{i}: n, l \in \mathbb{Z}, j=0, \ldots, M-1, i=1, \ldots, r\right\}$ is $\left\{D^{n} T_{M l} S^{-1} T_{j} \psi_{i}:\right.$ $n, l \in \mathbb{Z}, j=0, \ldots, M-1, i=1, \ldots, r\}$. It follows, by the proof of Theorem 1 , that $V_{0}(\Psi)$ is shift invariant by the lattice $M \mathbb{Z}$.

If $\Psi$ generates a Riesz basis, then conversely, the proof of Theorem 5 shows that if $V_{0}(\Psi)$ is shift invariant by the lattice $M \mathbb{Z}$, then the dual of the Riesz basis $\left\{D^{n} T_{M l} T_{j} \psi_{i}: n, l \in \mathbb{Z}, j=0, \ldots, M-1, i=1, \ldots, r\right\}$ has the form $\left\{D^{n} T_{M l} \phi_{j, i}: n, l \in \mathbb{Z}, j=0, \ldots, M-1, i=1, \ldots, r\right\}$.

As an immediate consequence of Proposition 2 we have the following corollaries.

Corollary 5. If $\Psi$ is a frame wavelet and $V_{J}(\Psi)$ is shift invariant, then the period of $\Psi$ divides $|A|^{J}$ for some $J \geq 0$.

Corollary 6. The period of a frame wavelet is 0 if $V_{0}(\Psi)$ is not shift invariant by any sublattice of $\mathbb{Z}$. The period of a Riesz wavelet is 0 if and only if $V_{0}(\Psi)$ is not shift invariant by any sublattice of $\mathbb{Z}$.

A less immediate consequence of Proposition 2 is the following result.

THEOREM 10. Suppose that $\Psi \subset L^{2}(\mathbb{R})$ is a Riesz wavelet associated with a dilation factor $A$ and $p_{1}, \ldots, p_{n}$ are all the prime factors of $A$. Then the period of $\Psi$ is either 0 or $\left(p_{1}\right)^{j_{1}} \ldots\left(p_{n}\right)^{j_{n}}$ for some integers $j_{1}, \ldots, j_{n} \geq 0$.

To show Theorem 10 we will use the following basic lemma.

Lemma 4. Let $n, m \in \mathbb{Z} \backslash\{0\}$ and $k=\operatorname{gcd}(n, m)$. Suppose that $W, V \subset$ $L^{2}(\mathbb{R})$ are closed, $W \subset V, W$ is $n \mathbb{Z}-S I$, and $V$ is $m \mathbb{Z}-S I$. Then the smallest $k \mathbb{Z}-S I$ space $S^{k \mathbb{Z}}(W)$ containing $W$ satisfies

$$
\mathcal{S}^{k \mathbb{Z}}(W):=\overline{\operatorname{span}}\left\{T_{z} f: z \in k \mathbb{Z}, f \in W\right\} \subset V .
$$

Proof. This is immediate. We decompose $k=a n+b m$ for some integers $a, b \in \mathbb{Z}$. Then for any $j \in \mathbb{Z}$,

$$
T_{j k}(W)=T_{j b m}\left(T_{j a n}(W)\right)=T_{j b m}(W) \subset T_{j b m}(V)=V .
$$

Proof of Theorem 10. Let $r \geq 1$ be the smallest integer such that $V_{0}(\Psi)$ is $r \mathbb{Z}$-SI. If there is no such $r$ then $V_{0}(\Psi)$ is not SI by any sublattice of $\mathbb{Z}$ and by Corollary 6 , the period of $\Psi$ is 0 , and we are done. 
Otherwise, we decompose $r=\left(p_{1}\right)^{j_{1}} \ldots\left(p_{n}\right)^{j_{n}} m$, where $j_{1}, \ldots, j_{n} \geq 0$ and $m$ is not divisible by any of $p_{1}, \ldots, p_{n}$. For any $j \leq-1$ we have $W_{j}(\Psi) \subset$ $V_{0}(\Psi)$, where $W_{j}(\Psi)$ is $|A|^{-j} \mathbb{Z}$-SI and $V_{0}(\Psi)$ is $r \mathbb{Z}$-SI. Thus, by Lemma 4 ,

$$
\mathcal{S}^{\left(p_{1}\right)^{j_{1}} \ldots\left(p_{n}\right)^{j_{n}} \mathbb{Z}}\left(W_{j}(\Psi)\right) \subset \mathcal{S}^{\operatorname{gcd}\left(|A|^{-j}, r\right) \mathbb{Z}}\left(W_{j}(\Psi)\right) \subset V_{0}(\Psi) \quad \text { for any } j \geq 1,
$$

since $\operatorname{gcd}\left(|A|^{-j}, r\right)$ must divide $\left(p_{1}\right)^{j_{1}} \ldots\left(p_{n}\right)^{j_{n}}$. Hence,

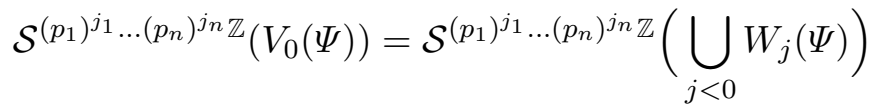

$$
\begin{aligned}
& =\overline{\sum_{j<0} \mathcal{S}^{\left(p_{1}\right)^{j_{1}} \ldots\left(p_{n}\right)^{j_{n}} \mathbb{Z}}\left(W_{j}(\Psi)\right)} \subset V_{0}(\Psi) .
\end{aligned}
$$

On the other hand, $V_{0}(\Psi) \subset \mathcal{S}^{\left(p_{1}\right)^{j_{1} \ldots}\left(p_{n}\right)^{j_{n}} \mathbb{Z}}\left(V_{0}(\Psi)\right)$, which shows by the minimality of $r$ that $m=1$ and $r=\left(p_{1}\right)^{j_{1}} \ldots\left(p_{n}\right)^{j_{n}}$.

By Proposition 2 and the minimality of $r$, the period of $\Psi$ is equal to $r$.

As an immediate corollary of Theorem 10 we obtain a partial answer to Daubechies and Han's question.

COROLlary 7. Suppose that $\Psi \subset L^{2}(\mathbb{R})$ is a Riesz wavelet associated with a prime dilation factor $A$. Then the period of $\Psi$ is either 0 or $|A|^{J}$ for some integer $J \geq 0$.

\section{Examples}

EXAmPLe 1. Suppose $a \geq 0$ and $\psi_{a} \in L^{2}(\mathbb{R})$ is given by

$$
\widehat{\psi}_{a}=\chi_{[-2 \pi,-\pi] \cup[\pi, 2 \pi]}+a \chi_{[-\pi,-\pi / 2] \cup[\pi / 2, \pi]} .
$$

The following table lists properties of $\psi_{a}$ as a function of a parameter $a$.

\begin{tabular}{cccc}
\hline Range of $a$ & Property of $\psi_{a}$ & Duals of $\psi_{a}$ & $V_{0}\left(\psi_{a}\right)$ \\
\hline$a=0$ & orthonormal wavelet & $\begin{array}{c}\text { canonical affine dual exists } \\
(=\text { orthonormal wavelet })\end{array}$ & SI \\
$0<a<1$ & biorthogonal Riesz wavelet & $\begin{array}{c}\text { canonical affine dual exists } \\
(=\text { biorthogonal Riesz wavelet })\end{array}$ & SI \\
& not a frame wavelet & no duals exist & SI \\
$a=1$ & frame wavelet (not Riesz $)$ & no affine duals exist & SI \\
\hline
\end{tabular}

The proof of the above properties is not always immediate, as Theorem 2 (ii) suggests. For example, to show that $V_{0}\left(\psi_{a}\right)$ is SI, it is necessary to prove that

$$
V_{0}\left(\psi_{a}\right)=\check{L}^{2}(-\pi, \pi) \quad \text { for all } a \geq 0 .
$$

The proof of this in the case of $1<a<\sqrt{2}$ is given by Theorem 2(ii). The exact same argument shows (22) also for $0<a \leq 1$. However, the case 
$a \geq \sqrt{2}$ requires a more general and less constructive approach that we will skip here.

If $a=0$ then $\psi_{a}$ is the orthonormal Shannon wavelet, hence all claimed properties follow. If $0<a<1$, then $\psi_{a}$ is a perturbation of the Shannon wavelet, which is a Riesz wavelet by the following variant of Lemma 1, to be found in [17, Theorem 5].

Lemma 5. Suppose $\mathcal{H}$ is a Hilbert space, $\left\{f_{i}\right\} \subset \mathcal{H}$ is a Riesz basis with constants $C_{1}$ and $C_{2}$, and $\left\{g_{i}\right\} \subset \mathcal{H}$ is a Bessel sequence with constant $C_{0}$. If $C_{0}<C_{1}$ then $\left\{f_{i}+g_{i}\right\}$ is a Riesz basis with constants $\left(\left(C_{1}\right)^{2}-\left(C_{0}\right)^{2}\right)^{1 / 2}$ and $\left(\left(C_{2}\right)^{2}+\left(C_{0}\right)^{2}\right)^{1 / 2}$.

Moreover, by Proposition 2, the period of $\psi_{a}$ is 1 , since $V_{0}\left(\psi_{a}\right)$ is $\mathbb{Z}$-SI. Therefore, $\psi_{a}$ is a biorthogonal Riesz wavelet for $0<a<1$ (see also $[21,22])$. Furthermore, $\psi_{a}$ is associated with the same MRA as the Shannon orthonormal wavelet (see $[6,7]$ ).

The fact that $\psi_{a}$ is not a frame wavelet for $a=1$ will follow from the corresponding property of $\psi_{b}$ in Example 2 for $b=\pi / 2$. Finally, the proof of Theorem 2(ii) shows that $\psi_{a}$ is a frame wavelet without affine duals for any $a>1$. Moreover, $\psi_{a}$ is not a Riesz wavelet. Otherwise, $\psi_{a}$ would need to be a biorthogonal Riesz wavelet since $V_{0}\left(\psi_{a}\right)$ is $\mathbb{Z}$-SI. This is a contradiction with the fact that $\psi_{a}$ has no affine duals for $a>1$.

ExAmple 2. Suppose $0 \leq b \leq 2 \pi$ and $\psi_{b} \in L^{2}(\mathbb{R})$ is given by

$$
\widehat{\psi}_{b}=\chi_{[-2 \pi,-b] \cup[b, 2 \pi]} \text {. }
$$

The following table lists properties of $\psi_{b}$ as a function of a parameter $b$.

\begin{tabular}{cccc}
\hline Range of $b$ & Property of $\psi_{b}$ & Duals of $\psi_{b}$ & $V_{0}\left(\psi_{b}\right)$ \\
\hline$b=0$ & not a frame wavelet & no duals exist & not SI \\
$0<b \leq \pi / 4$ & frame wavelet (not Riesz) & no affine duals exist & SI \\
$\pi / 3<b<2 \pi / 3$ & not a frame wavelet & no duals exist & SI \\
$2 \pi / 3 \leq b<\pi$ & biorthogonal Riesz wavelet & canonical affine dual exists & SI \\
& & (= biorthogonal Riesz wavelet) & \\
$b=\pi$ & orthonormal wavelet & canonical affine dual exists & SI \\
& & (= orthonormal wavelet) & \\
$\pi<b \leq 2 \pi$ & not a frame wavelet & no duals exist & SI \\
\hline
\end{tabular}

Despite the simplicity of the form of $\psi_{b}$, the justifications of the above properties are even more involved than those in Example 1. We start by studying the properties of $V_{0}\left(\psi_{b}\right)$.

It is clear that for any $0 \leq b \leq 2 \pi$ we have

$$
V_{0}\left(\psi_{b}\right) \subset \check{L}^{2}(-\pi, \pi) .
$$


Moreover, $V_{0}\left(\psi_{b}\right) \neq \check{L}^{2}(-\pi, \pi)$ for $b=0$, since $f \in L^{2}(\mathbb{R})$ given by $\widehat{f}=$ $\chi_{(-\pi, 0)}-\chi_{(0, \pi)}$ satisfies $f \perp W_{j}\left(\psi_{b}\right)$ for $j<0$ and hence $f \perp V_{0}\left(\psi_{b}\right)$. Therefore, $V_{0}\left(\psi_{b}\right)$ is not SI for $b=0$. Using a rather involved orthogonality argument, which we will skip here, it is possible to show that

$$
V_{0}\left(\psi_{b}\right)=\check{L}^{2}(-\pi, \pi) \quad \text { for } 0<b \leq \pi .
$$

Furthermore, it is easy to show that

$$
V_{0}\left(\psi_{b}\right)=\check{L}^{2}\left(\bigcup_{j<0}\left(-2^{j+1} \pi,-2^{j} b\right) \cup\left(2^{j} b, 2^{j+1} \pi\right)\right) .
$$

Therefore, $V_{0}\left(\psi_{b}\right)$ is SI for every $0<b \leq 2 \pi$.

Now we turn to frame properties of the affine system $\left\{D^{n} T_{l} \psi_{b}\right\}$. It is clear that $\left\{D^{n} T_{l} \psi_{b}\right\}$ is not even a Bessel sequence for $b=0$. Hence, we can move to the situation when $b>0$ by considering different ranges of the parameter $b$.

CASE I. If $0<b \leq \pi / 4$, then $\psi^{0}$, given by $\widehat{\psi}^{0}=\chi_{(-\pi, b) \cup(b, \pi)}$, is a frame wavelet with the lower frame constant $\geq 2$. Therefore, by Lemma $1, \psi_{b}=$ $\psi^{0}+\psi^{1}$ is also a frame wavelet, where $\psi^{1}$ is the Shannon wavelet, i.e., $\widehat{\psi}^{1}=$ $\chi_{(-2 \pi,-\pi) \cup(\pi, 2 \pi)}$. A similar argument to that in the proof of Theorem 2(ii) shows that $\psi_{b}$ does not have any affine duals.

Interestingly, if $\pi / 4<b \leq \pi / 3$ then the above argument fails, since $\psi^{0}$ as above is a frame wavelet with the lower frame constant 1 , and we cannot conclude that $\psi_{b}=\psi^{0}+\psi^{1}$ generates a frame. However, based on an heuristic evidence we believe that $\psi_{b}$ is still a frame wavelet for $\pi / 4<b \leq \pi / 3$, but our present arguments are overly complicated, and hence we skip them here. It appears that the borderline case $b=\pi / 3$ is the hardest and it would be interesting to have a rigorous and direct proof of this case.

CASE II. Next, we will show that $\psi_{b}$ is not a frame wavelet for every $\pi / 3<b<2 \pi / 3$. To show this we need to use the following standard identity, which can be shown by the periodization argument:

$$
\sum_{z \in \mathbb{Z}}\left|\left\langle f, T_{z} \psi_{b}\right\rangle\right|^{2}=\frac{1}{2 \pi} \int_{0}^{2 \pi}\left|\sum_{k \in \mathbb{Z}} \widehat{f}(\xi+2 \pi k) \overline{\widehat{\psi}_{b}(\xi+2 \pi k)}\right|^{2} d \xi
$$

for any $f \in L^{2}(\mathbb{R})$. Let $K_{b}=(b, 2 \pi / 3) \cup(2 \pi / 3+b, 4 \pi / 3)$. Then by a simple calculation

$$
\sum_{z \in \mathbb{Z}}\left|\left\langle f, T_{z} \psi_{b}\right\rangle\right|^{2}=\frac{1}{2 \pi} \int_{K_{b}}|\widehat{f}(\xi-2 \pi)+\widehat{f}(\xi)|^{2} d \xi \quad \text { for any } f \in \check{L}^{2}\left(L_{b}\right),
$$

where

$$
L_{b}=(-\infty,-2 \pi) \cup\left(K_{b}-2 \pi\right) \cup(-b, b) \cup K_{b} \cup(2 \pi, \infty) .
$$


For any natural number $n$ define a function $f_{n} \in L^{2}(\mathbb{R})$ by

$$
\widehat{f}_{n}=\sum_{k=0}^{n}\left[\chi_{\left(2^{-k} \pi / 3-\varepsilon, 2^{-k} \pi / 3\right)}-\chi_{\left(-2^{-k} \pi / 3-\varepsilon,-2^{-k} \pi / 3\right)}\right],
$$

where $\varepsilon=\varepsilon(n)>0$ is sufficiently small. Intuitively, one should think of $\widehat{f}_{n}$ as a linear combination of point masses $\sum_{k=0}^{n}\left(\delta_{2^{-k} \pi / 3}-\delta_{-2^{-k} \pi / 3}\right)$. We claim that

$$
D^{j} f_{n} \in \check{L}^{2}\left(L_{b}\right) \quad \text { for all } j \in \mathbb{Z} \text {. }
$$

Indeed, (25) follows immediately from

$$
\begin{aligned}
2^{j}\left(\left(\frac{2^{-k} \pi}{3}-\varepsilon, \frac{2^{-k} \pi}{3}\right)\right. & \left.\cup\left(-\frac{2^{-k} \pi}{3}-\varepsilon,-\frac{2^{-k} \pi}{3}\right)\right) \\
& \subset \begin{cases}(-b, b), & j \leq k, \\
\left(K_{b}-2 \pi\right) \cup K_{b}, & j=k+1, k+2, \\
(-\infty, 2 \pi) \cup(2 \pi, \infty), & j \geq k+3,\end{cases}
\end{aligned}
$$

where $k=0, \ldots, n$ and $0<\varepsilon=\varepsilon(n)<2^{-n-2} \min \{b-\pi / 3,2 \pi / 3-b\}$.

Let $S$ be the frame operator corresponding to the affine system generated by $\psi_{b}$. Note that by Theorem $4, S$ is bounded from above. Our goal is to show that $S$ is not bounded from below. Combining (23)-(25) we have

$$
\begin{aligned}
\left\|S f_{n}\right\|^{2} & =\sum_{j \in \mathbb{Z}} \sum_{z \in \mathbb{Z}}\left|\left\langle f_{n}, D^{j} T_{z} \psi_{b}\right\rangle\right|^{2}=\sum_{j \in \mathbb{Z}} \sum_{z \in \mathbb{Z}}\left|\left\langle D^{j} f_{n}, T_{z} \psi_{b}\right\rangle\right|^{2} \\
& =\frac{1}{2 \pi} \sum_{j=1}^{n+2} 2^{-j} \int_{K_{b}}\left|\widehat{f}_{n}\left(2^{-j}(\xi-2 \pi)\right)+\widehat{f}_{n}\left(2^{-j} \xi\right)\right|^{2} d \xi=\frac{2 \varepsilon}{\pi} .
\end{aligned}
$$

Here, we used the fact that for $\xi \in K_{b}$,

$$
\begin{aligned}
\widehat{f}_{n}\left(2^{-j}(\xi-2 \pi)\right)+\widehat{f}_{n}\left(2^{-j} \xi\right) & \\
\quad= & \begin{cases}\chi_{(2 \pi / 3-2 \varepsilon, 2 \pi / 3)}(\xi)-\chi_{(-2 \pi / 3-2 \varepsilon,-2 \pi / 3)}(\xi), & j=1, \\
0, & j=2, \ldots, n+1, \\
\chi_{\left(4 \pi / 3-2^{n+2} \varepsilon, 4 \pi / 3\right)}(\xi)-\chi_{\left(-4 \pi / 3-2^{n+2} \varepsilon,-4 \pi / 3\right)}(\xi), & j=n+2 .\end{cases}
\end{aligned}
$$

On the other hand,

$$
\left\|f_{n}\right\|^{2}=\frac{1}{2 \pi}\left\|\widehat{f}_{n}\right\|^{2}=\frac{\varepsilon(n+1)}{\pi} .
$$

Since $n$ is arbitrary, this shows that the frame operator $S$ is not bounded from below.

Despite its technicality, the above argument has a very simple idea behind it involving cancellation of masses at pairs of points $(-4 \pi / 3,2 \pi / 3)$ and $(-2 \pi / 3,4 \pi / 3)$. To see this, it helps to think of $\widehat{\psi}_{b}$ as $\delta_{-4 \pi / 3}+\delta_{-2 \pi / 3}+\delta_{2 \pi / 3}+$ $\delta_{4 \pi / 3}$, and of $\widehat{f}_{n}$ as $\sum_{k=0}^{n}\left(\delta_{2^{-k} \pi / 3}-\delta_{-2^{-k} \pi / 3}\right)$. 
CASE III. If $2 \pi / 3 \leq b<\pi$, then $\psi_{b}$ is a biorthogonal Riesz wavelet. Indeed, we will first show that $\psi_{b}$ has a dual frame wavelet $\phi_{b}$ given by

$$
\widehat{\phi}_{b}=\chi_{(-2 \pi,-\pi) \cup(\pi, 2 \pi)}-\chi_{(-4 \pi+2 b,-2 \pi) \cup(2 \pi, 4 \pi-2 b)} \text {. }
$$

By Theorem 4, $\phi_{b}$ generates a Bessel sequence. By the well-known characterization of dual affine systems, it suffices to check that

$$
\begin{gathered}
\sum_{j \in \mathbb{Z}} \widehat{\psi_{b}}\left(2^{j} \xi\right) \overline{\widehat{\phi}_{b}\left(2^{j} \xi\right)}=1 \quad \text { for a.e. } \xi \\
t_{q}(\xi)=\sum_{j=0}^{\infty} \widehat{\psi_{b}}\left(2^{j} \xi\right) \overline{\widehat{\phi}_{b}\left(2^{j}(\xi+2 \pi q)\right)}=0 \quad \text { for a.e. } \xi \text { and for } q \text { odd. }
\end{gathered}
$$

It is immediate that (26) holds. To show (27) it suffices to consider only the case when $|\xi|<2 \pi$ by looking at the support of $\widehat{\psi}_{b}$. Likewise, since $\operatorname{supp} \widehat{\psi}_{b} \cap\left(\operatorname{supp} \widehat{\phi}_{b}+2 \pi k\right)=\emptyset$ for $|k| \geq 3$, we can assume that $q= \pm 1$ and that the sum in (27) reduces only to the terms $j=0$ and $j=1$.

If $\pi<|\xi|<2 \pi$ then the sum (27) reduces only to the term $j=0$, which is 0 , since

$$
\widehat{\phi}_{b}(\xi+2 \pi q)=0 \quad \text { for } \pi<|\xi|<2 \pi \text { and } q \text { odd. }
$$

Likewise, if $\pi / 2<|\xi|<\pi$ then the sum (27) reduces only to the terms $j=0$ and $j=1$,

$$
t_{q}(\xi)=\sum_{j=0}^{1} \widehat{\psi_{b}}\left(2^{j} \xi\right) \widehat{\widehat{\phi}_{b}\left(2^{j}(\xi+2 \pi q)\right)} .
$$

An easy argument shows that the only way of obtaining non-zero terms is when either $b<\xi<\pi$ and $q=-1$ or $-\pi<\xi<-b$ and $q=1$, in which case $t_{q}(\xi)=1 \cdot 1+1 \cdot(-1)=0$. If $\pi / 4<|\xi|<\pi / 2$ then the sum (27) reduces only to $j=1$ by looking at the support of $\widehat{\psi}_{b}$. In this case, it is immediate that $t_{q}(\xi)=0$. Finally, if $|\xi|<\pi / 4$ then it is also obvious that all terms in (27) must be 0 . This proves that $\phi_{b}$ is an affine dual of $\psi_{b}$.

Next, we will show that the affine frames generated by $\psi_{b}$ and $\phi_{b}$ are biorthogonal, i.e.,

$$
\left\langle D^{j} T_{z} \psi_{b}, D^{j^{\prime}} T_{z^{\prime}} \phi_{b}\right\rangle=\delta_{j, j^{\prime}} \delta_{z, z^{\prime}} \quad \text { for all } j, j^{\prime}, z, z^{\prime} \in \mathbb{Z} .
$$

By [19, Section 3.1], (28) is equivalent to

$$
\sum_{k \in \mathbb{Z}} \widehat{\psi}_{b}(\xi+2 \pi k) \overline{\widehat{\phi}_{b}\left(2^{j}(\xi+2 \pi k)\right)}=\delta_{j, 0} \quad \text { for a.e. } \xi \text { and } j \geq 0 \text {. }
$$

Since the expression in (29) is $2 \pi$-periodic, it suffices to verify (29) for a.e. $\xi \in(-2 \pi,-\pi) \cup(\pi, 2 \pi)$. It is immediate that (29) holds for $j=0$. Next, suppose that $\xi \in(\pi, 2 \pi)$ and $j=1$. Then the sum in (29) reduces to 


$$
\begin{aligned}
\widehat{\psi}_{b}(\xi-2 \pi) \overline{\widehat{\phi}_{b}(2(\xi-2 \pi))} & +\widehat{\psi}_{b}(\xi) \overline{\widehat{\phi}_{b}(2 \xi)} \\
= & \chi_{(-\pi,-b)}(\xi-2 \pi) \cdot 1-1 \cdot \chi_{(2 \pi, 4 \pi-2 b)}(2 \xi)=0 .
\end{aligned}
$$

By inspecting the supports of $\widehat{\psi}_{b}$ and $\widehat{\phi}_{b}$, it is also clear that if $j \geq 2$ then all products in (29) vanish. A similar argument works for $\xi \in(-2 \pi,-\pi)$, which shows (29) and consequently (28). Therefore, $\psi_{b}$ and $\phi_{b}$ form a pair of biorthogonal Riesz wavelets. Since $\psi_{b}$ is associated with the same MRA as the Shannon orthonormal wavelet (see $[6,7]$ ), $\psi_{b}$ and $\phi_{b}$ are biorthogonal MRA Riesz wavelets.

CAsE IV. The case when $\pi \leq b \leq 2 \pi$ is trivial. If $b=\pi$ then $\psi_{b}$ is the Shannon orthonormal wavelet. If $\pi<b \leq 2 \pi$ then the affine system generated by $\psi_{b}$ is not complete in $L^{2}(\mathbb{R})$, hence it is not a frame. This completes the discussion of Example 2.

EXAMPLE 3. Let $\psi$ be a Meyer orthonormal wavelet, chosen to have smooth Fourier transform and $\operatorname{supp} \widehat{\psi}=(-8 \pi / 3,-2 \pi / 3) \cup(2 \pi / 3,8 \pi / 3)$ (see [19, Section 3.4]). We claim that the frame wavelet $D^{-1} \psi$ has no dual of the form $\left\{D^{n} T_{l} \phi\right\}$; the computation is as below. Notice, however, that $\eta=D^{-2} \psi$ is a tight frame wavelet, since $\widehat{\eta}(\xi)=2 \widehat{\psi}(4 \xi)$, which is supported in the set $(-2 \pi / 3,-\pi / 6) \cup(\pi / 6,2 \pi / 3)$. Thus,

$$
\begin{gathered}
\sum_{j \in \mathbb{Z}}\left|\widehat{\eta}\left(2^{j} \xi\right)\right|^{2}=4 \quad \text { for a.e. } \xi \\
\sum_{j \geq 0} \widehat{\eta}\left(2^{j} \xi\right) \widehat{\eta}\left(2^{j}(\xi+2 \pi q)\right)=0 \quad \text { for a.e. } \xi \text { and for } q \text { odd. }
\end{gathered}
$$

Again, let $\psi$ be a Meyer orthonormal wavelet; there exists an affine frame $\left\{D^{n} T_{l} \psi_{i}: i=1,2\right\}$ with no affine duals but with $V_{0}\left(\psi_{1}, \psi_{2}\right)$ shift invariant.

Define $\psi_{1}$ and $\psi_{2}$ by

$$
\begin{aligned}
\widehat{\psi}_{1}(\xi) & =\widehat{\psi}(2 \xi) \\
\widehat{\psi}_{2}(\xi) & =\widehat{\psi}(\xi) \chi_{(-4 \pi / 3,-2 \pi / 3)}(\xi) .
\end{aligned}
$$

Note that $\left\{D^{n} T_{l} \psi_{1}\right\}$ is a frame and $\left\{D^{n} T_{l} \psi_{2}\right\}$ is a Bessel sequence, so $\Psi=$ $\left\{\psi_{1}, \psi_{2}\right\}$ is a frame wavelet.

We first claim that this frame wavelet has no duals of the form $\left\{D^{n} T_{l} \phi_{i}\right.$ : $i=1,2\}$. Suppose to the contrary that there is a frame wavelet $\Phi=\left\{\phi_{1}, \phi_{2}\right\}$ which is a dual to $\Psi$. We must have the following:

$$
\begin{gathered}
\sum_{i=1}^{2} \sum_{j \in \mathbb{Z}} \widehat{\psi}_{i}\left(2^{j} \xi\right) \overline{\widehat{\phi}_{i}\left(2^{j} \xi\right)}=1 \quad \text { for a.e. } \xi \\
\sum_{i=1}^{2} \sum_{j=0}^{\infty} \widehat{\psi}_{i}\left(2^{j} \xi\right) \overline{\widehat{\phi}_{i}\left(2^{j}(\xi+2 \pi q)\right)}=0 \quad \text { for a.e. } \xi \text { and for } q \text { odd. }
\end{gathered}
$$


For $\xi \in(2 \pi / 3,4 \pi / 3),(33)$ reduces to

$$
\widehat{\psi}_{1}(\xi) \overline{\widehat{\phi}_{1}(\xi+2 \pi q)}=0 \text { for a.e. } \xi
$$

$q$ odd. Setting $q=-1$ yields that $\widehat{\phi}_{1}(\xi)=0$ for $\xi \in(-4 \pi / 3,-2 \pi / 3)$. Therefore, for $\xi \in(-2 \pi / 3,-\pi / 3)$, (32) reduces to

$$
\begin{aligned}
1 & =\widehat{\psi}_{1}(\xi) \overline{\widehat{\phi}_{1}(\xi)}+\widehat{\psi}_{1}(2 \xi) \overline{\widehat{\phi}_{1}(2 \xi)}+\widehat{\psi}_{2}(2 \xi) \overline{\widehat{\phi}_{2}(2 \xi)}=\widehat{\psi}_{1}(\xi) \overline{\widehat{\phi}_{1}(\xi)}+\widehat{\psi}_{2}(2 \xi) \overline{\widehat{\phi}_{2}(2 \xi)} \\
& =\widehat{\psi}_{1}(\xi)\left[\overline{\widehat{\phi}_{1}(\xi)}+\overline{\widehat{\phi}_{2}(2 \xi)}\right] .
\end{aligned}
$$

Therefore, since $\widehat{\psi}_{1}(\xi)$ decays to 0 on $(-2 \pi / 3,-\pi / 3), \overline{\widehat{\phi}_{1}(\xi)}+\overline{\widehat{\phi}_{2}(2 \xi)}$ must be unbounded, which is a contradiction to the fact that $\Phi$ was a frame wavelet.

We next claim that $V_{0}(\Psi)$ is shift invariant. It is easy to see that $\overline{\operatorname{span}}\left\{T_{z} \psi_{i}: z \in \mathbb{Z}, i=1,2\right\}=\check{L}^{2}((-4 \pi / 3,-\pi / 3) \cup(\pi / 3,4 \pi / 3))$. Therefore, $V_{0}(\Psi)=\check{L}^{2}((-2 \pi / 3,2 \pi / 3))$, which is clearly shift invariant.

\section{References}

[1] L. Baggett, H. Medina, and K. Merrill, Generalized multiresolution analyses, and a construction procedure for all wavelet sets in $\mathbb{R}^{n}$, J. Fourier Anal. Appl. 5 (1999), $563-573$.

[2] J. Benedetto and $\mathrm{S} . \mathrm{Li}$, The theory of multiresolution analysis frames and applications to filter banks, Appl. Comput. Harmon. Anal. 5 (1998), 389-427.

[3] M. Bownik, A characterization of affine dual frames in $L^{2}\left(\mathbb{R}^{n}\right)$, ibid. 8 (2000), 203-221.

[4] - The structure of shift-invariant subspaces of $L\left(\mathbb{R}^{n}\right)$, J. Funct. Anal. 177 (2000), 282-309.

[5] -, On characterizations of multiwavelets in $L\left(\mathbb{R}^{n}\right)$, Proc. Amer. Math. Soc. 129 (2001), 3265-3274.

[6] -, Riesz wavelets and generalized multiresolution analyses, Appl. Comput. Harmon. Anal. 14 (2003), 181-194.

[7] M. Bownik and G. Garrigós, Biorthogonal wavelets, MRA's and shift-invariant spaces, Studia Math., to appear.

[8] R. Catalan, Oversampling and preservation of tightness in affine frames, Proc. Amer. Math. Soc. 130 (2002), 1031-1034.

[9] O. Christensen, Moment problems and stability results for frames with applications to irregular sampling and Gabor frames, Appl. Comput. Harmon. Anal. 3 (1996), $82-86$.

[10] C. Chui, W. Czaja, M. Maggioni, and G. Weiss, Characterization of general tight wavelet frames with matrix dilations and tightness preserving oversampling, J. Fourier Anal. Appl. 8 (2002), 173-200.

[11] C. Chui and X. Shi, $n \times$ oversampling preserves any tight affine frame for odd $n$, Proc. Amer. Math. Soc. 121 (1994), 511-517.

[12] C. Chui, X. Shi, and J. Stöckler, Affine frames, quasi-affine frames, and their duals, Adv. Comput. Math. 8 (1998), 1-17.

[13] A. Cohen, I. Daubechies, and J.-C. Feauveau, Biorthogonal bases of compactly supported wavelets, Comm. Pure Appl. Math. 45 (1992), 485-560. 
[14] X. Dai and D. Larson, Wandering vectors for unitary systems and orthogonal wavelets, Mem. Amer. Math. Soc. 640 (1998).

[15] I. Daubechies and B. Han, The canonical dual frame of a wavelet frame, Appl. Comput. Harmon. Anal. 12 (2002), 269-285.

[16] I. Daubechies, B. Han, A. Ron, and Z. Shen, Framelets: MRA-based constructions of wavelet frames, ibid. 14 (2003), 1-46.

[17] S. J. Favier and R. A. Zalik, On the stability of frames and Riesz bases, ibid. 2 (1995), 160-173.

[18] E. Hernández, D. Labate, and G. Weiss, A unified characterization of reproducing systems generated by a finite family, II, J. Geom. Anal. 12 (2002), 615-662.

[19] E. Hernández and G. Weiss, A First Course on Wavelets, CRC Press, Boca Raton, FL, 1996.

[20] M. Holschneider, Wavelets. An Analysis Tool, Clarendon Press, Oxford, 1995.

[21] H. O. Kim, R. Y. Kim, and J. K. Lim, Characterizations of biorthogonal wavelets which are associated with biorthogonal multiresolution analyses, Appl. Comput. Harmon. Anal. 11 (2001), 263-272.

[22] D. Larson, W. S. Tang, and E. Weber, Multiwavelets associated with countable abelian groups of unitary operators in Hilbert spaces, preprint, 2001.

[23] R. Laugesen, Translational averaging for completeness, characterization and oversampling of wavelets, Collect. Math. 53 (2002), 211-249.

[24] A. Ron and Z. Shen, Affine systems in $L_{2}\left(\mathbb{R}^{d}\right)$ : the analysis of the analysis operator, J. Funct. Anal. 148 (1997), 408-447.

[25] - - - Affine systems in $L^{2}\left(\mathbb{R}^{d}\right) I I$ : dual systems, J. Fourier Anal. Appl. 3 (1997), 617-637.

[26] E. Weber, On the translation invariance of wavelet subspaces, ibid. 6 (2000), 551558.

Department of Mathematics

University of Michigan

Ann Arbor, MI 48109-1109, U.S.A.

E-mail: marbow@umich.edu

Department of Mathematics

University of Wyoming

Current addres of M. Bownik:

Department of Mathematics

University of Oregon

Eugene, OR 97403-1222, U.S.A.

E-mail: mbownik@uoregon.edu

Laramie, WY 82071-3036, U.S.A.

E-mail: esw@uwyo.edu

Current addres of E. Weber:

Department of Mathematics

Iowa State University

Ames, IA 50011-2064, U.S.A.

Received January 17, 2003

Revised version June 17, 2003 